\title{
Cyclic variability of the circumstellar disk of the Be star $\zeta$ Tauri
}

\section{Long-term monitoring observations ${ }^{\star}$}

\author{
S. Štefl ${ }^{1}$, Th. Rivinius ${ }^{1}$, A. C. Carciofi ${ }^{2}$, J.-B. Le Bouquin ${ }^{1}$, D. Baade ${ }^{3}$, K. S. Bjorkman ${ }^{4, \star \star}$, E. Hesselbach ${ }^{4, \star \star}$, \\ C. A. Hummel ${ }^{3}$, A. T. Okazaki ${ }^{5}$, E. Pollmann ${ }^{6}$, F. Rantakyrö ${ }^{7}$, and J. P. Wisniewski ${ }^{8, \star \star}$ \\ ${ }^{1}$ European Organisation for Astronomical Research in the Southern Hemisphere, Casilla 19001, Santiago 19, Chile \\ e-mail: sstefl@eso.org \\ 2 Instituto de Astronomia, Geofísica e Ciências Atmosféricas, Universidade de São Paulo, Rua do Matão 1226, Cidade Universitária, \\ São Paulo, SP 05508-900, Brazil \\ 3 European Organisation for Astronomical Research in the Southern Hemisphere, Karl-Schwarzschild-Str. 2, \\ 85748 Garching bei München, Germany \\ ${ }^{4}$ University of Toledo, Department of Physics \& Astronomy, MS111 2801 W. Bancroft Street Toledo, OH 43606, USA \\ 5 Faculty of Engineering, Hokkai-Gakuen University, Toyohira-ku, Sapporo 062-8605, Japan \\ 6 Emil-Nolde-Str.12, 51375 Leverkusen, Germany \\ 7 Gemini Observatory, Southern Operations Center, c/o AURA, Casilla 603, La Serena, Chile \\ 8 NSF Astronomy \& Astrophysics Postdoctoral Fellow, Department of Astronomy, University of Washington, Box 351580, Seattle, \\ WA 98195, USA
}

Received 23 December 2008 / Accepted 1 July 2009

\begin{abstract}
Context. Emission lines formed in decretion disks of Be stars often undergo long-term cyclic variations, especially in the violet-to-red $(V / R)$ ratio of their primary components. The underlying structural and dynamical variations of the disks are only partly understood. From observations of the bright Be-shell star $\zeta$ Tau, the possibly broadest and longest data set illustrating the prototype of this behaviour was compiled from our own and archival observations. It comprises optical and infrared spectra, broad-band polarimetry, and interferometric observations.

Aims. The dense, long-time monitoring permits a better separation of repetitive and ephemeral variations. The broad wavelength coverage includes lines formed under different physical conditions, i.e. different locations in the disk, so that the dynamics can be probed throughout much of the disk. Polarimetry and interferometry constrain the spatial structure. All together, the objective is a better understand the dynamics and life cycle of decretion disks.

Methods. Standard methods of data acquisition, reduction, and analysis were applied.

Results. From $3 V / R$ cycles between 1997 and 2008, a mean cycle length in $\mathrm{H} \alpha$ of 1400-1430 days was derived. After each minimum in $V / R$, the shell absorption weakens and splits into two components, leading to 3 emission peaks. This phase may make the strongest contribution to the variability in cycle length. There is no obvious connection between the $V / R$ cycle and the 133-day orbital period of the not otherwise detected companion. $V / R$ curves of different lines are shifted in phase. Lines formed on average closer to the central star are ahead of the others. The shell absorption lines fall into 2 categories differing in line width, ionization/excitation potential, and variability of the equivalent width. They seem to form in separate regions of the disk, probably crossing the line of sight at different times. The interferometry has resolved the continuum and the line emission in Bry and HeI 2.06. The phasing of the Br $\gamma$ emission shows that the photocenter of the line-emitting region lies within the plane of the disk but is offset from the continuum source. The plane of the disk is constant throughout the observed $V / R$ cycles. The observations lay the foundation for the fully self-consistent, one-armed, disk-oscillation model developed in Paper II.
\end{abstract}

Key words. stars: circumstellar matter - stars: emission line, Be - stars: individual: $\zeta$ Tauri - infrared: stars - polarization stars: early type

\section{Introduction}

Circumstellar disks of classical Be-stars have been known for decades as the place of origin of their characteristic Balmer

\footnotetext{
^ Based partly on observations collected at the European Southern Observatory, Chile (Prop. Nos. 073.D-0234, 074.D-0240, 078.D-0542, and 081.D-2005; as well as archival data from programs 074.D-0573 and 076.B-0055).

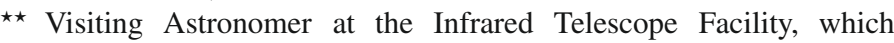
is operated by the University of Hawaii under cooperative agreement NNX08AE38A with the National Aeronautics and Space Administration, Science Mission Directorate, Planetary Astronomy Program.
}

emission lines. Polarimetric (McLean \& Brown 1978) and combined interferometric and spectro-polarimetric observations (Quirrenbach et al. 1997; Vakili et al. 1998) have brought the long discussion of the geometry of the disks to a definitive conclusion. These observations confirm directly that the disks are geometrically thin with low opening angles of about 5-15 degrees. The outer radius of the $\mathrm{H} \alpha$ emission was estimated at typically 10-20 stellar radii.

The detailed dynamics of Be-star disks, however, is not as clearly known. Various hypotheses exist, but the observations have not so far yielded fully unambiguous results. The relations between (a) stellar $v$ sin $i$ (Hanuschik 1989) and (b) disk size 
(Quirrenbach et al. 1997), and separation of the emission peaks, details of circumstellar line shapes (Hummel \& Vrancken 2000), and the occurrence of central quasi-emission peaks in shell stars (Rivinius et al. 1999) are all consistent with Kepler-like rotation, in which the rotation velocity varies as $r^{-j}$, where $r$ is the distance from the star. For a strictly Keplerian disk with circular orbits the exponent $j$ is equal to 0.5 . Analyses of spectral line profiles (Hummel \& Vrancken 2000) suggests that $j<0.65$.

Hanuschik (1994, 2000) and Waters \& Marlborough (1994) showed that any radially outward directed velocities in the disk must be lower than a few $\mathrm{km} \mathrm{s}^{-1}$. However, the angular momentum in a Keplerian disk increases with $r^{1 / 2}$, Be disks are formed from matter outflowing from the star, and at least part of the disk eventually escapes from the star's gravity. Therefore, there must be a net angular momentum transfer from the star to the disk and then outwards through the disk. The mechanism for this momentum transfer, albeit not yet conclusively identified, is most likely related to dynamical viscosity. A viscous decretion disk model (Lee et al. 1991; Porter 1996; Okazaki 2001) has recently been successfully applied to the circumstellar disk of the Be star $\delta$ Sco (Carciofi et al. 2006) and, at present, is the strongest candidate to describe the structure of Be-star disks.

Purely circular Keplerian disks are also unable to explain the complex variability of the Balmer emission lines, notably the so-called $V / R$ (the violet-to-red flux ratio of the emission peaks) variations. Most observations of $V / R$ variations concern lower Balmer lines, but they can also be seen in virtually all other emission lines. Often, $V / R$ variations are cyclic with timescales of 5-10 years (Okazaki 1991). The amplitudes can be large to spectacular. Nevertheless, many $V / R$ cycles look strongly perturbed because of concomitant large variations in the emissionline strength relative to the continuum and, presumably, the unsteady character of the mass loss from the central star.

The first models for the $V / R$ variations, a precessing elliptical ring (Huang 1973), spheroidal/ellipsoidal variable-mass loss (Doazan 1987), and a variable stellar wind causing expansions and contractions of the disk (Mennickent \& Vogt 1991) were not physically self-consistent. They were finally made obsolete by Okazaki's model of one-armed density oscillations in the disk (Okazaki 1991, 1997). It is based on work by Kato (1983), who showed that the only possible global oscillation mode in an often thin Keplerian disk has $m=1$. The idea of density waves was developed further by Papaloizou et al. (1992), Okazaki (2000), and Papaloizou \& Savonije (2006). A few scattered interferometric observations detected decentered density enhancements in circumstellar disks of $\zeta$ Tau (Vakili et al. 1998) and $\gamma$ Cas (Berio et al. 1999).

The wide acceptance of the model is based mainly on qualitative comparisons. Its detailed application to specific sets of $V / R$ curves and emission line profiles has been pending to date. A strong reason is the poor repetitiveness of $V / R$ cycles, which makes it difficult to decide what the model can be expected to reproduce. Furthermore, the typically long duration of the cycles has kept the statistics at a low level. Also, detailed comparisons between model and observations have only become feasible with the advent of three-dimensional radiative transfer codes (Carciofi \& Bjorkman 2006). The generic theoretical modeling combining the dynamics of $m=1$ disk oscillations with a radiative-transfer code was successfully pioneered by Hummel \& Hanuschik (1997).

Štefl et al. (2007) analyzed the $V / R$ variations in five Be-shell stars with a companion star. They find that in some stars the $V / R$ variability is phase-locked to the orbital motions. In others it is not. A peculiarity seemingly limited to binary Be-shell stars (but not exhibited by all of them) is the appearance of triple-peak $\mathrm{H} \alpha$ emission profiles (which may also be described by a doubling of the self-absorption in the disk). $\zeta$ Tau is one of the examples presented by Štefl et al. (2007). The occurrence of such profiles seems restricted to a narrow phase interval at the beginning of the transition from $V \ll R$ to $V \sim R$. They are not reproduced by present versions of the disk-oscillation model.

The star $\zeta$ Tau (123 Tau, HR 1910, HD 37202 ; B2 IV) is among the most suitable targets for an in-depth observational and theoretical test of the disk-oscillation model. The present emission state, which started in 1990 (Guo et al. 1995, their Fig. 2), shows very stable $V / R$ variations with a cycle length of about 1500 days (Rivinius et al. 2006). Compared to other $V / R$-variable Be stars, secular and ephemeral variations have recently (see below) been small enough that observations obtained in different cycles can be fairly safely assembled into one picture.

For decades, $\zeta$ Tau has been known as a spectroscopic binary. A comprehensive set of radial velocity measurements was compiled and analyzed by Harmanec (1984), who derived a period of $132.9735 \mathrm{~d}$.

Being a bright object reachable from both northern and southern latitudes, $\zeta$ Tau is one of the most observed $\mathrm{Be}$ stars. At the distance of 417 light years (corresponding to the Hipparcos parallax of 7.82 mas) not only the circumstellar disk can be resolved with present-day interferometers but also spectro-interferometry extends this resolution to the disk dynamics. The limb-darkened photospheric diameter is estimated at 0.4 mas (see e.g. Tycner et al. 2004; Gies et al. 2007). At the Hipparcos distance, this corresponds to a radius of 5.5 to $6 R_{\odot}$.

The major axis of the $\mathrm{H} \alpha$ emitting disk was measured by Quirrenbach et al. (1997) to 4.53 mas, corresponding to a radius of $11.3 R_{\star}$ and by Tycner et al. to 3.14 mas (almost $8 R_{\star}$ ). Gies et al. (2007) derived the major axis of 1.99 mas (about $5 R_{\star}$ ) for the $K$-band continuum emitting disk. These values were derived from the full width at half maximum (FWHM) of a Gaussian fit. The differences can be reconciled by assuming that the disk was less fully developed during the later observations as is also suggested by a lower $\mathrm{H} \alpha$ emission strength (see below). In the $K$-band continuum, Gies et al. measured the semi-major axis of the disk to 1.79 mas or $4.5 R_{\star}$ (Gaussian FWHM). All these values are lower than the estimated Roche-lobe radius for the primary of the system, which Tycner et al. compute as 5.3 mas $\left(26.5 R_{\star}\right)$ at a binary separation of 9.2 mas $\left(46 R_{\star}\right)$.

This first paper of the series summarizes the extended observational material (see Sect. 2) and focuses on a detailed phenomenological description of the variability corresponding to the $V / R$ cycle. The variations in the visible part of the spectrum are analyzed in Sect. 3. The variability in emission lines in the $J H K$-bands is described in Sect. 4. Section 5 is devoted to the VLTI/AMBER spectro-interferometry and contemporaneous optical and IR spectroscopy, and Sect. 6 deals with new and previously published polarimetric observations. The last two sections discuss and summarize the observational results.

The second paper (Carciofi et al., this volume) presents a detailed self-consistent physical model of the observations. It combines the 2D global oscillation model of Okazaki (1997) and the $3 \mathrm{D}$ radiative-transfer code HDUST of (Carciofi \& Bjorkman 2006, 2008). 
Table 1. Spectroscopic datasets in the visual range.

\begin{tabular}{|c|c|c|c|c|c|c|c|c|}
\hline $\begin{array}{l}\text { Data } \\
\text { set }\end{array}$ & $\begin{array}{l}\text { Observing } \\
\text { season }\end{array}$ & $\begin{array}{c}\text { JD } \\
2400000+\end{array}$ & Telescope & Instrument & $\begin{array}{l}\text { No. of } \\
\text { spectra }\end{array}$ & $\begin{array}{c}\text { Resolving } \\
\text { power }\end{array}$ & $\begin{array}{c}\text { Spectral } \\
\text { range }[\AA]]\end{array}$ & Ref. \\
\hline $\mathrm{A}$ & 1991 & 48347 & Heidelberg $0.9 \mathrm{~m}$ & FLASH & 1 & 20000 & $4050-6780$ & 0 \\
\hline B & 1992-2007 & $49049-51486$ & Ondřejov $2 \mathrm{~m}$ & slit spectr. & 43 & 8500 & $6300-6700$ & 1 \\
\hline $\mathrm{C}$ & $1994 \& 2000$ & $49592 \& 51572$ & OHP $1.93 \mathrm{~m}$ & ElODIE & 3 & 45000 & $3850-6800$ & 2 \\
\hline D & $2000-2003$ & $51045-52726$ & Ondřejov 2 m & HEROS & 23 & 20000 & $3700-8600$ & 1 \\
\hline $\mathrm{E}$ & 2005-2008 & $53399-54714$ & ESO/MPI $2.2 \mathrm{~m}$ & FEROS & 25 & 48000 & $3700-9000$ & 1,0 \\
\hline $\mathrm{F}$ & $2000-2008$ & $51850-54174$ & Schmidt-Cassegrain & Slitless-Grating & 129 & 14000 & $6500-6700$ & 3 \\
\hline $\mathrm{G}$ & 2006-2007 & $54005-54515$ & SCT $0.3 \mathrm{~m}$ & LHIRES & 12 & 13425 & $6520-6700$ & 4 \\
\hline $\mathrm{H}$ & 2006 & $54387 \& 54083$ & Celestron 11 & LHIRES & 3 & 17000 & $6520-6620$ & 5 \\
\hline I & 2006 & $54169 \& 54200$ & Takahashi TSC225 & LHIRES & 7 & 17000 & $3000-7000$ & 6 \\
\hline $\mathrm{J}$ & $2007-2008$ & $54162-54550$ & Schmidt-Cassegrain & Slitless-Grating & 32 & 14000 & $6500-6700$ & 7 \\
\hline K & $2007 \& 2008$ & $54442 \& 54527$ & Newton & LHIRES & 2 & 7000 & $6490-6700$ & 8 \\
\hline $\mathrm{L}$ & 2008 & 54532 & Celestron C14 & LHIRES & 2 & 17000 & $6520-6610$ & 9 \\
\hline M & $2007-2008$ & $54406-54515$ & Meade LX200 & LHIRES & 10 & 17000 & $6520-6610$ & 10 \\
\hline $\mathrm{N}$ & 2008 & $54106-54359$ & $\mathrm{C} 11$ & LHIRES & 3 & 17000 & $4000-8000$ & 11 \\
\hline $\mathrm{O}$ & 2007 & 54331 & OHP ED120 & LHIRES & 1 & 17000 & $6400-6800$ & 12 \\
\hline $\mathrm{P}$ & 2006-2008 & $54021-54714$ & $\mathrm{ESO} / \mathrm{MPG} 2.2 \mathrm{~m}$ & FEROS & 18 & 48000 & 3700-9000 & 0 \\
\hline Q & 1999-2007 & $51489-54433$ & Ritter 1 m & echelle & 61 & 26000 & $4600-6700$ & 0 \\
\hline $\mathrm{R}$ & 2007 & 54384 & OPD/LNA & ECASS & 1 & 16000 & $6500-6620$ & 0 \\
\hline
\end{tabular}

References: 1 - Rivinius et al. (2006); 2 - BeSS,observer: C. Neiner; 3.- E. Pollmann, data used in Pollmann \& Rivinius (2008), private communication; 4 - BeSS observer: B. Mauclaire; 5 - BeSS observer: C. Buil; 6 - BeSS observer: E. Barbotin; 7 - BeSS observer: E. Pollmann; 8 - BeSS observer: J. Guarro Fló; 9 - BeSS observer: J. Ribeiro; 10 - BeSS observer: J.-N. Terry; 11 - BeSS observer: O. Thizy; 12 - BeSS observer: V. Desnoux; 0 - this paper.

\section{Observations}

\subsection{Visual spectroscopy}

In order to maximize the coverage of the $V / R \mathrm{H} \alpha$ cycles, new observations were combined with data from the literature, the ESO Science Archive, and from the BeSS database (see below). Table 1 summarizes the spectroscopic data sets used for this study. The description of the HEROS and FEROS spectrographs can be found in Kaufer (1998) and Kaufer et al. (1999), respectively. The FLASH instrument is an earlier version of the HEROS spectrograph with only one spectral arm (covering the range from 4050 to $6700 \AA$ ) and a somewhat lower resolution due to the use of a larger fiber, which determines the effective slit width.

The OPD/LNA observation was made with a Cassegrain spectrograph, equipped with a 1200 grooves/mm grating blazed at $6562 \AA$ and a $1024 \times 1024$ pixel CCD. Observations at the Ritter observatory were done with an echelle spectrograph in the Cassegrain focus of the 1-m telescope, using the old camera equipped with a $1200 \times 800$ pixel CCD (cf. Sect. 2.4).

In recent years, the technological progress has brought bright stars like $\zeta$ Tau within reach of quite a few amateur telescopes and spectrographs. Spectra of many Be stars are regularly deposited in, and conveniently available from, BeSS (http:// basebe.obspm. fr). As Table 1 shows, they account for a large fraction of the total optical spectroscopy used in this paper.

\subsection{Infrared spectroscopy}

Fourteen infrared (IR) spectra were obtained between March 5, 2004 and October 9, 2007 using the SpeX spectrograph at the 3.0-m NASA Infrared Telescope Facility (IRTF) on Mauna Kea (Rayner et al. 2006). With a 0' $3 \times 15^{\prime \prime}$.0 slit in the shortwavelength cross-dispersed (SXD) observing mode, a resolving power of $R \sim 2000$ spectroscopy was achieved from 0.8 to $2.4 \mu \mathrm{m}$. The observing and data reduction techniques mirrored those used in previous SpeX programs to study classical Be stars (see, e.g., Wisniewski 2005; Wisniewski et al. 2007). Observations of $\zeta$ Tau were immediately followed by observations of a nearby A0V star located at a similar air mass, to facilitate optimal telluric correction (Vacca et al. 2003), along with a series of quartz-tungsten-halogen flat field and argon arc lamp exposures. The IDL-based Spextool software was used to perform the basic data reduction and spectral extraction using the techniques described in Cushing et al. (2004).

\subsection{Interferometry}

Our near-IR interferometric observations with AMBER (Petrov et al. 2007) and three 8-m telescopes (UT 1, 2, and 4) of ESO's VLTI interferometer were made during the night of December 12, 2006. Interferometric fringes from all 3 pairs of telescopes (hereafter called baselines) were measured across the $K$-band, at medium spectral resolution of $R \approx 1500$. Because fringe tracking was not supported at the time, it was not possible to properly record the full wavelength range. The observations were, therefore, taken around the $\operatorname{Br} \gamma(2.16 \mu \mathrm{m})$ and $\mathrm{He} \mathrm{I}(2.06 \mu \mathrm{m})$ lines. The $\zeta$ Tau observations were interlaced with observations of the calibration stars HD 39699 and HD 59 686, for which diameters of 1.03 mas and 1.30 mas, respectively (Mérand et al. 2005), were adopted.

In principle, three distinct quantities can be extracted for each spectral channel of the AMBER spectro-interferometer: the fringe visibility (measuring the spatial extension of the emitting material), the fringe phase (measuring the position of emitting material), and the closure-phase (the sum of the 3 phases obtained for the 3 interferometric arms, sensitive to asymmetries in the source brightness distribution). In AMBER data sets, the signal-to-noise ratio on the closure-phase is very low and only visibilities and phases were used for the analysis.

The data reduction was done using the amdlib-2.2 package, which employs the P2VM algorithm (Tatulli et al. 2007). In 
a first step, the instrumental calibration matrix with the standard calibration files provided by ESO was computed. This is mandatory in order to properly convert the raw individual frames into raw interferometric visibilities and phases (about thousand measurements per observation). The next steps consist of averaging these different frames into individual measurements, and performing the final calibration. Based on our past experience of spectro-interferometry with AMBER, two different strategies were adopted. In the following they are distinguished as absolute reduction and differential reduction. Only a brief description is included here, as both methods are now considered to be standard data reduction steps for AMBER.

Absolute data reduction: The objective of this method is to provide an absolutely calibrated estimation of the fringe visibility (the fringe phase cannot be determined in an absolute manner). It requires the multiplicative effect of the atmospheric turbulence (generally called transfer function) to be properly estimated.

- For each observation, the top $20 \%$ of the frames with the best signal-to-noise ratio were averaged. This ratio provides the most stable transfer function, see discussion in Tatulli et al. (2007) and Millour et al. (2007).

- The transfer function was estimated by averaging all observations of the calibration stars. Their scatter is a measure for the uncertainty of the transfer function. The resulting stability is very poor, with temporal fluctuations as large as $30 \%$, partially due to the atmospheric turbulence and to the Unit Telescope infrastructures, which generate non-stationary vibrations.

- Absolutely calibrated quantities were derived by dividing separately in all spectral channels - the raw visibilities of $\zeta$ Tau by the derived transfer function. Error bars on the transfer function completely dominate the final uncertainties.

- Finally, all spectral channels were averaged (avoiding the $\mathrm{He} \mathrm{I}$ and $\mathrm{Br} \gamma$ lines) to provide a single visibility across the band for each observation. Formally it introduces a small amount of wavelength smearing, which is however negligible at the level of precision.

It is worth noting that this observation strategy has been optimized for differential interferometry, not for absolute calibration of the fringe visibility. Only a single calibration star has been observed for each AMBER instrument setup, and the observations of the calibration stars do not bracket properly the observations of the science target. Therefore it is hard to asses the reliability of the absolute calibration (usually done by comparing values obtained using different calibration stars). Consequently, the fringe visibility derived with this method should be interpreted carefully.

Differential data reduction: This method aims mainly at a precise measurement of the differential quantities (visibility and phase) in the $\operatorname{Br} \gamma$ and $\mathrm{He}$ I lines with respect to the adjacent continuum. Compared with the previous method, a significantly higher SNR can be obtained because the atmosphere affects all spectral channels in very nearly the same way. The main drawback is that the continuum level is obviously lost.

- First, as many as possible individual frames were averaged. To do so, all consecutive observations with the same instrument setup were merged together before averaging the $70 \%$ of the frames with the best signal-to-noise. It was tested that this method provides the best final accuracy on the differential quantities.

- The here presented AMBER data were corrupted by ripples with low beat frequency in the spectral direction (few cycles along the complete $K$-band spectrum ${ }^{1}$ ). These artifacts mainly affect the phases and not the visibilities, explaining why they are not of a big concern regarding the absolute data reduction. To correct them, a high-pass filter along the spectral direction was applied to the phases. Different methods (high-pass filter, optimal filtering at the corrupted frequencies, manual fit of the continuum ripples) were tested but differences in the resulting $\mathrm{He} \mathrm{I}$ and $\mathrm{Br} \gamma$ phase profiles could be seen.

- At that stage, the continuum levels for both the phase and the visibility are arbitrary. The brightness distribution of the $K$-band continuum emitting region has already been constrained by Gies et al. (2007). Because they used longer baselines and more precise absolute calibration, their results are significantly better than the AMBER measurements for the continuum (see previous paragraph). Therefore the AMBER continuum level around the $\mathrm{Br} \gamma$ and $\mathrm{He}$ I lines was forced to to match the CHARA model of Gies et al. (2007), which is displayed in Fig. 10 .

\subsection{Polarimetry}

The polarimetric data are from observations made with the Halfwave Polarimeter (HPOL, Nordsieck \& Harris 1996). The instrument was used primarily on the 0.9-m telescope at the Pine Bluff Observatory (PBO), operated by the University of Wisconsin (UW). The data are available through the HPOL web site (http://www.sal.wisc.edu/HPOL) developed by M.R. Meade and B.L. Babler, and data prior to 1995 are also available through the NASA MAST archive. The database includes a wide range of hot stars, the polarimetric variability of which has been discussed in Bjorkman \& Meade (2005).

Data from 1989-1994 were obtained using a dual Reticon array detector, which provided spectropolarimetry over a wavelength range of 3200-7600 , with a spectral resolution of $25 \AA$ (Wolff et al. 1996). In 1995, the HPOL detector was upgraded to a $400 \times 1200$ pixel CCD camera, and data since then have covered a more extended wavelength range from 3200-10500 $\AA$, with an improved spectral resolution of $7 \AA$ below $6000 \AA$ and $10 \AA$ above this point (Nordsieck \& Harris 1996). For further details about HPOL, see Nook (1990) and Wolff et al. (1996). As described by Wolff et al. (1996), the data were processed and analyzed using REDUCE, a specialized spectropolarimetric software package developed at UW. Instrumental polarization is monitored regularly as part of the observing program at PBO, and all data are fully calibrated for instrumental effects to an accuracy of $0.025 \%$ in the $V$ band.

For the purposes of this study of $\zeta$ Tau, the spectropolarimetric data were binned to approximate broad-band $U B V R I$ results. The individual uncertainty of an HPOL measurement was estimated by a statistics over a series of data of polarized standards: It is about $0.01 \%$ for the polarization degree and $1 \mathrm{deg}$ for the polarization angle in $B V R I$, and about two to three times as high in the $U$-band.

1 The interferences between the light beams reflected inside the
AMBER polarizers created "Moire fringes" in the spectral direction.
The optics responsible for these artifacts was identified and properly replaced in October 2008. 


\section{Visual spectral line variability}

\subsection{Long-term evolution of the $\zeta$ Tau circumstellar disk and its $V / R$ variations}

Harmanec (1984, his Fig. 2) showed that the present wellpronounced $V / R$ variations were absent in earlier decades: At least from 1920 to about 1950 there was no $V / R$ cyclicity. A $V / R$-variable phase started in the mid-1950s and lasted for about three cycles until 1980. From 1980 to 1990 the star was again stable with $V \approx R$. From 1980 to 1985 the equivalent width (EW) of the $\mathrm{H} \alpha$ emission decreased from about -23 to $-12 \AA$ while the star brightened by 0.3 to 0.4 magnitudes in the $U B V$ passbands, also getting slightly bluer. This behavior is typical of Be-shell stars as described observationally by Harmanec (1983) and predicted before by Poeckert \& Marlborough (1978, their Fig. 33) for the case of decreasing base density of the disk.

From 1985 to 1990 the disk was in a low-density state (Guo et al. 1995, their Figs. 2 and 3). The $V / R$ variability was not well sampled observationally. But short-term (days) variability probably dominated (Guo et al. 1995, their Fig. 5). It would, then, have resembled a phase observed in $\mu$ Cen by Hanuschik et al. (1993), in which rapid $V / R$ variations were due mainly to discrete mass loss events and the subsequent circularization of the ejected matter.

Around 1990, the emission strength began to increase again to a high level of almost $-30 \AA$, together with brightness and colour changes as expected for an edge-on disk with increasing base density. At the same time, the disk entered a phase of $V / R$ variations. Since this replenishing of the disk, the emission lines became weaker ( $\mathrm{H} \alpha \mathrm{EW}$ between -15 and $-20 \AA$ ), but the $V / R$ variations are still ongoing. The AAVSO database ${ }^{2}$ indicates that the magnitude was stable over the last 8 years $\left(\left\langle m_{V}\right\rangle \sim 3.02 \pm\right.$ 0.07).

\subsection{Variations of the total $\mathrm{H} \alpha$ equivalent width}

Equivalent widths were measured by means of an automatic MIDAS (Grosbøl \& Ponz 1990) procedure in the interval 6546$6581 \AA$ A. Except for a few outliers, all total EWs between 1992 and 2008 are in the interval -11.5 to $-20 \AA$, with a mean of $(-15.5 \pm 0.2) \AA$. After an initial increase of emission lasting from about 1991 to 1994 the value remained rather stable. This is a further indicator of the validity of the assumption that the individual cycles are comparable to each other.

In view of the uncertainties in the normalization of the echelle spectra with imperfectly corrected wiggles and systematic differences between spectrographs with very different spectral resolution, a more detailed analysis of the measured total equivalent widths and their temporal variations was not attempted.

\subsection{Shell absorption lines}

In the visual (and higher-resolution) spectra, two types of shell lines (dubbed "narrow-" and "broad-line group", NLG and BLG) and their associated cyclic behavior can be distinguished. Pure NLG-type shell absorption is found in lines of Fe II (near-UV lines only), Fe III, Ni II, O I, Na I, and He I. The cores of the Balmer lines, too, fall into the NLG category. Pure BLG-type shell absorption is seen in some lines of Si II, Fe II (visual

\footnotetext{
2 American Association of Variable Star Observers; http: //www . aavso.org/data
}

Table 2. Properties of individual $V / R$ cycles.

\begin{tabular}{cccccc}
\hline \hline $\begin{array}{c}\text { Cycle } \\
\text { number }\end{array}$ & \multicolumn{2}{c}{ Max. $V / R$} & Cycle & \multicolumn{2}{c}{ Triple peak } \\
JD 24 & $V / R$ & length [d] & [JD 24 range] & [d] \\
\hline I & 50414 & 1.62 & 1419 & $51137-51440$ & 303 \\
II & 51833 & 1.58 & 1527 & $52580-53094$ & 494 \\
III & 53360 & 1.44 & 1230 & $54005-54208$ & 203 \\
IV & 54590 & 1.40 & N/A & N/A-N/A & NA \\
\hline
\end{tabular}

wavelengths), Cr II, Ti II, Mg I, and O I. This group also includes Mg II 4481, which is among the easiest lines to excite in a Be circumstellar environment. The NLG/BLG classification of the most important spectral lines in the optical spectrum is marked in Table 3.

The assignment of a line to BLG or NLG is not in all cases and not at all epochs unique. For instance, Fe II 5137 and Si II 6347 normally show the BLG characteristics, but may have additional NLG components in certain phases of the $V / R$ cycle. Other BLG lines, too, exhibit additional temporary NLG components, especially around $V / R$ phase $\tau \approx 0.25$ (see Sect. 3.5). Typical representatives are the strongest shell lines due to Si II, Ca II, Ni II, and Fe II.

Besides the obvious property of their widths, the two groups also differ in their variations of radial velocity and symmetry as described in Sect. 7.7

\subsection{Length of the V/R cycles}

Some confusion may occur due to different definitions of the $V / R$ ratio in the literature. They differ in the reference flux level, with respect to which the peak maxima are measured. This paper uses the more common definition $\left(V / R=F_{V} / F_{R}\right.$, where $F_{V}$ and $F_{R}$ are the relative fluxes at the violet and red emission peak maxima, respectively), according to which $V / R$ is computed without prior correction for the underlying continuum level. Therefore, the results are less influenced by the spectrum normalization than in the other definition, which uses only the flux above the continuum level. Consequently, this approach enables to combine reasonably well $V / R$ values even in heterogeneous databases. Since the photometric variability has been small (Sect. 3.1), while the $\mathrm{H} \alpha$ line emission was strong, the continuum variability should have little effect on the $V / R$ values measured in this way. The $V / R$ values obtained for the interval 1993-2008 are visualized in Fig. 1.

The observations before JD 2450500 are insufficient to conclude whether the $V / R$ cycle was not yet stabilized or whether the sampling was too sparse to deduce a more regular $V / R$ variability. However, the subsequent observations define nicely three complete cycles marked I, II, and III in Fig. 1. Cycle IV just commenced about JD 2454590 . Starting Julian Dates and lengths of the cycles are summarized in Table 2 . The cycle-averaged $V / R$ curve is displayed in Fig. 2.

The mean $V / R$ curve is smooth and roughly symmetric except for some time shortly after the minimum. During this ascending branch, the $\mathrm{H} \alpha$ emission is split into three peaks (or the self-absorption is split into 2 components), making the definition of "the" $V$ and "the" $R$ component ambiguous (see Sect. 3.5.4 and Fig. 6). An increased scatter in $V / R$ values is the result (see Figs. 1 and 2). Obviously, the disk variability cannot be characterized by a single parameter such as $V / R$ alone.

By contrast, the $V / R$ maxima are very well defined and can be used as fiducial marks for measuring the length of $V / R$ cycles. 

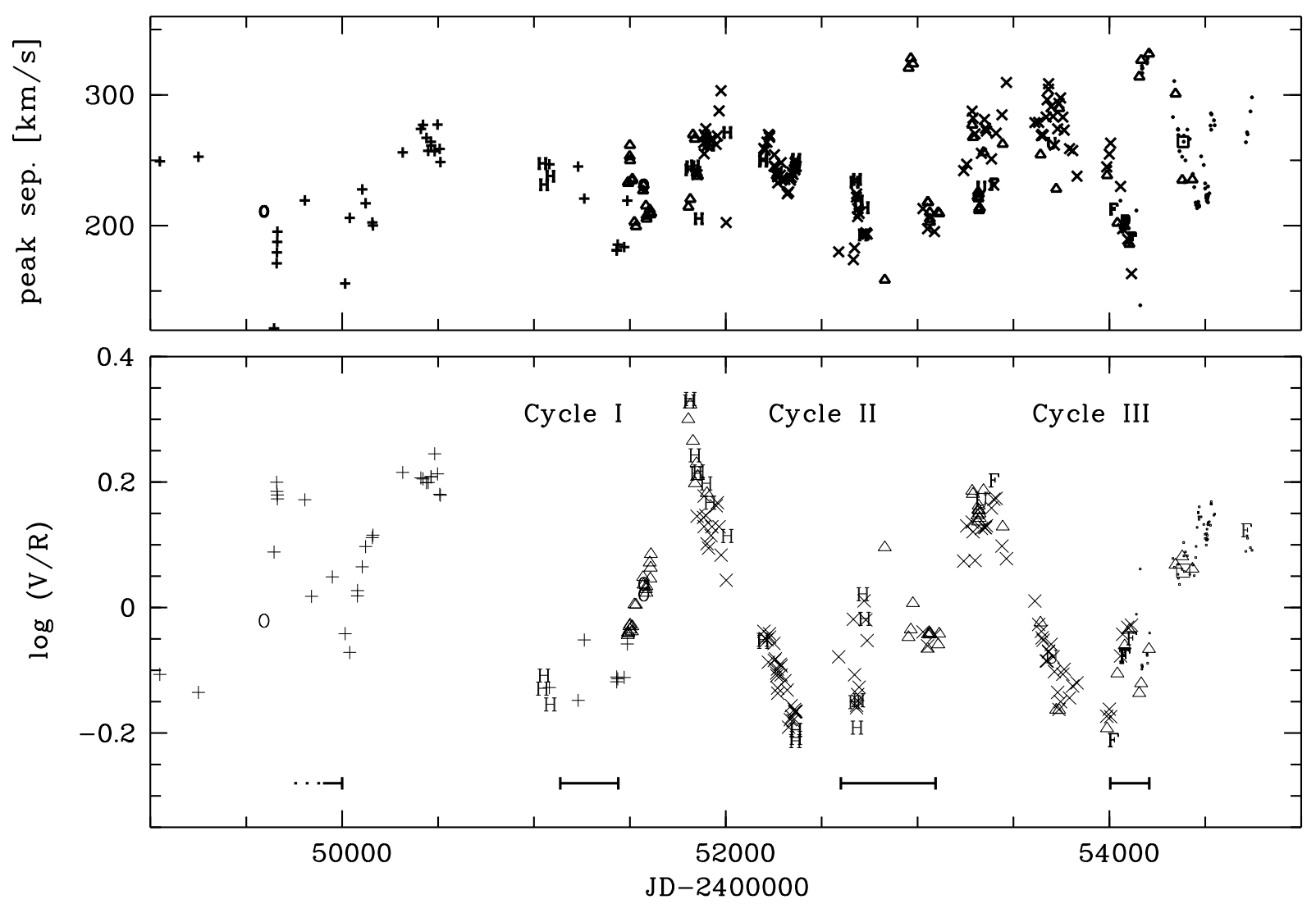

Fig. 1. $\mathrm{H} \alpha V / R$ variations (lower panel) and emission peak separation (upper panel) of $\zeta$ Tau in the period 1992-2008 showing the detailed character of more than $3 V / R$ cycles since 1996. The symbols correspond to the following data sets: (+) Ondrejov slit spectrograph, (O) OHP $1.93 \mathrm{~m},(\times)$ Pollmann, (H) HERos, (F) FERos, $(\triangle)$ Ritter Observatory, ( $)$ BeSS database, $(\square)$ A. Carciofi, see also Table 1. Phases with triplepeaked emission line profiles are indicated by solid horizontal bars.

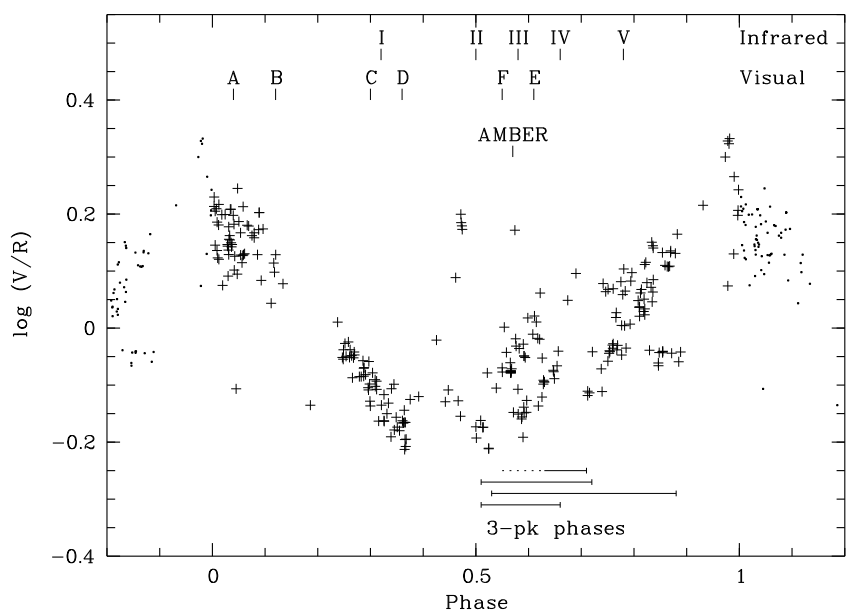

Fig. 2. $V / R$ data from Fig. 1, phase binned with $J D 50414+1429 \times E$. Horizontal lines indicate the triple-peak phases (3-pk) from Table 2 , which introduce a higher scatter in the corresponding phase interval because they make the definition of the $V$ and $R$ emission components doubtful. The letters in the upper part of the figure mark the phases of the AMBER observation and of repesentative visual and infrared spectra used in Sects. 3.5 and 4 (see also Tables 4 and 5).

Fourth-order polynomials fitted to the peaks over different baselines indicate an error in their position of 5-8 days.

In spite of the systematic uncertainty in $V / R$, Fig. 1 seems to suggests a secondary $V / R$ maximum that coincides with the triple-peak phases and follows the main minimum. Apparently, such secondary maxima in the cyclic $V / R$ variations were not so far reported for any Be star. Their height and widths vary from cycle to cycle and the strongest secondary peak can be recognized in Cycle II at JD 2452 700-2 453000.

A formal Fourier frequency analysis was only applied to $\mathrm{H} \alpha V / R$ values not affected by a third component (see Table 2 for the corresponding dates). Such a restriction is suggested by the sensitivity of most period-search methods to non-sinusoidal perturbations and to a lower accuracy of the $V / R$ values during the triple-peak phase. The CLEAN method of the sinusoid fitting (Kaufer et al. 1996) gives periods $P_{1}=(685 \pm 5) \mathrm{d}$ and $P_{2}=(1405 \pm 26)$ d, obviously the former one is the harmonic produced due to variable cycle lengths. Scargle's method (Scargle 1982) is less likely to return harmonics and suggests 1429 days.

This mean duration of the $V / R$ cycles is shorter than the values derived by Pollmann \& Rivinius (2008), 1475 days, and by Rivinius et al. (2006), 1503 days. The differences are well explained by the different data sets used. In particular, the earlier studies could not include the shorter-than-average triple-peak phase of Cycle III. Even more different and variable cycle length can be identified during the $1955-1980 \mathrm{~V} / \mathrm{R}$ variable phase. The compiled data presented by Harmanec (1984) show three maxima, defining -in the same sense as in this paper- two complete cycles of about 2290 and 1290 days.

Radial-velocity (RV) measurements suffer from the inhomogeneity of the database in a similar fashion as the absolute emission strength does. The impact of this inhomogeneity is reduced in the RV separation of the red and violet emission peaks 
Table 3. Selected spectral lines in the optical spectrum and their classification into broad-line (BLG) and narrow-line group (NLG), respectively, as described in Sect. 3.5.

\begin{tabular}{lcl}
\hline Wavelength & Ion & Comment \\
\hline 3187.746 & He I & NLG, no phot. \\
3465.642 & Ni II & NLG \\
3468.678 & Fe II & NLG \\
3471.386 & Ni II & NLG \\
3513.987 & Ni II & NLG, strong \\
3530.505 & He I & pure phot. \\
3554.412 & He I & pure phot. \\
3587.268 & He I & pure phot. \\
3634.238 & He I & pure phot. \\
3968.469 & Ca II & BLG + NLG \\
3994.997 & N II & pure phot. \\
4002.592 & Si II & BLG \\
4067.031 & Ni II & BLG + NLG \\
4128.054 & Si II & BLG + NLG \\
4130.872 & Si II & BLG + NLG \\
4258.154 & Fe II & BLG \\
4294.099 & Ti II & BLG \\
4481.126 & Mg II & BLG \\
4824.127 & Cr II & BLG \\
5015.678 & He I & NLG + weak phot. \\
5100.727 & Fe II & BLG \\
5127.387 & Fe III & NLG \\
5156.111 & Fe III & NLG \\
5169.033 & Fe II & BLG + NLG \\
5172.684 & Mg I & BLG \\
5183.604 & Mg I & BLG \\
5197.577 & Fe II & BLG + NLG \\
5226.543 & Ti II & BLG \\
5316.615 & Fe II & BLG + NLG \\
5679.558 & N II & pure phot. \\
5875.625 & He I & NLG + phot. \\
5889.951 & Na I & NLG \\
5895.924 & Na I & NLG \\
6147.741 & Fe II & BLG \\
6149.258 & Fe II & BLG \\
6156.755 & O I & BLG \\
6158.187 & O I & BLG \\
6347.109 & Si II & BLG + NLG \\
6371.371 & Si II & BLG + NLG \\
6678.154 & He I & NLG + phot. \\
7774.166 & O I & NLG \\
\hline & &
\end{tabular}

(Fig. 1). The separation follows the $V / R$ cycle, but with a possible phase shift and a considerably higher scatter, which not surprisingly is most prominent when the triple-peak profiles are present. The dates of maxima of peak separation are delayed by 100-200 days with respect to the $V / R$ maxima.

\subsection{Spectral evolution during the $V / R$ cycle}

The repeatability of the $V / R$ cycles for more than a decade justifies the introduction of the concept of an ephemeris and a phase so that observations can be compared between cycles. In the following, all phases refer to the formal cycle length of 1429 days from above and adopt the $V / R$ maximum of JD 2450414 as the reference epoch. The co-phased $V / R$ curve is given in Fig. 2.

In order to more completely characterize the disk variability than the $V / R$ ratio can do, 6 optical reference spectra were selected. Their $V / R$ phases are marked in Fig. 2. The spectra were sorted such that the phase of equal peak height in presumably optically thin lines like Fe II 6318 has just finished in spectrum "F" and is about to return after spectrum " $E$ ". (Note that in Fig. 2 "F" appears at an earlier phase than " $E$ ". But this is only an artifact of the co-phasing of cycles with different length. Phenomenologically, " $F$ " is actually following " $E$ ".)

This classification is restricted to the visual spectral range. For the IR spectra, which are more limited in phase coverage and spectral resolution, a separate scheme is introduced in Sect. 4.

The following subsections describe the main optical $V / R$ phases and their transitions. The definition of the $V / R$ phases is for $\mathrm{H} \alpha$. The variability of other lines may have significant offsets in phase, as detailed below.

\subsection{1. $V>R$}

This phase is represented by the "A" profiles in Fig. 3. In all emission lines the violet peak is higher than the red one. The shell absorption lines weaken and become narrower. Both NLG and BLG are red-shifted with respect to the systemic velocity. However, there are differences: The red edge of BLG shell absorption is very steep, and the blue edge rather asymptotically joins the continuum. In the NLG, it is the blue edge which is steeper than the red one. All ionic species are present but some low-ionization lines begin to weaken.

\subsection{2. $V=R$, descending to $V<R$, and deep absorption}

When the strength of the $R$ peak in $\mathrm{H} \alpha$ begins to approach the one of the $V$ peak, the peaks in $\mathrm{H} \beta$ are already of almost equal height, and Fe II lines even exhibit an inverted $V / R$ ratio (cf. spectra "B" and "C" in Fig. 3).

Some of the low-ionization shell lines of the BLG category have completely vanished, while the NLG shell lines are at maximum depth now. The Balmer series is maximally visible up to $\mathrm{H} 40 / 41$. At other phases, the limit is reached around H34/H35 (see Fig. 7).

While pure BLG lines such as Mg II 4481 are slightly blueshifted, pure NLG lines reside at zero velocity and are very narrow. Also shortwards of the Balmer discontinuity several shell lines are far more clearly present than at other phases, phenomenologically belonging to the NLG. Their FWHM is only about 17 to $20 \mathrm{~km} \mathrm{~s}^{-1}$, i.e. about the thermal width.

Shell lines of Fe II and Si II, however, develop a twocomponent structure related to both groups: a very narrow and deep absorption core at zero velocity is superimposed on a broad and shallower absorption that has its own distinct core farther to the blue. The two cores can be clearly identified as parts of the BLG and NLG characteristics, respectively.

\subsection{3. $V<R$}

The "D" profiles in Fig. 3 illustrate this phase. The red peak reaches its maximal strength. Both NLG and BLG lines are blue-displaced with respect to the systemic velocity. The dual NLG/BLG characteristics of some lines weakens and finally vanishes in that NLG cores disappear while the remainder of the profile evolves as if belonging to the BLG.

\subsection{4. $V=R$, ascending to $V>R$, and triple-peak structure}

Spectra "E" and "F" in Fig. 3 offer a general overview of this phase, and Fig. 6 illustrates the temporal evolution in $\mathrm{H} \alpha$. The most intriguing property of this phase is that the $\mathrm{H} \alpha$ line 

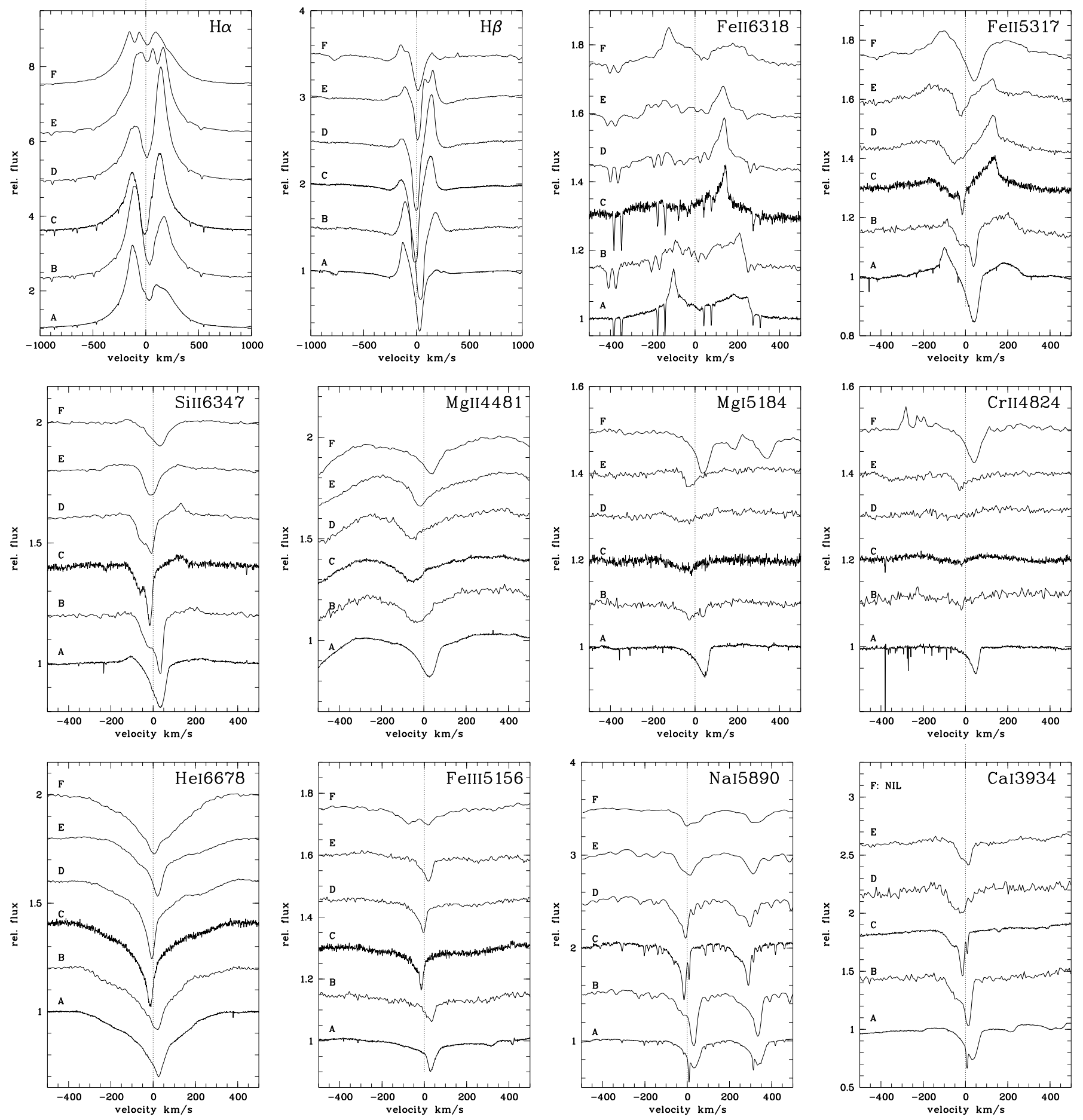

Fig. 3. Evolution of representative lines in the visible spectrum during the $V / R$ cycle. See Table 4 for the properties of spectra "A" to "F". The H $\alpha$ $V / R$ can bbe read off Fig. 2.

emission strongly deviates from the classical Be star profile in that it no longer shows clear double peaks with a wellpronounced central depression. Rather, the central depression is filled in (or split) and three peaks of similar strength co-exist. Although the central depression does not get filled in in the other Balmer lines, and they do not show multiple peaks, their structure is more complex than in other phases.

The O I 8446 line, which is excited by fluorescence of $\operatorname{Ly} \beta$ and therefore usually assumed to present an optically thin tracer of the hydrogen distribution, does not show any sign of a triple peak (see Fig. 4). Instead, the profile is double-peaked with a central depression. Noteworthy is that the radial velocity of the red peak of the O I profile coincides with the local minimum in the triple peaked $\mathrm{H} \alpha$ and $\mathrm{H} \beta$ profiles.

The shell absorption lines are strong and yet relatively broad. Lowly ionized species like Ti II, Cr II, or Mg II prevail, but highionization species like Fe III still produce noticeable absorption.

Spectrum "F" (Fig. 3) was obtained in 1991. Since then, the shell spectrum has generally weakened. But there is no doubt that spectrum " $F$ " is representative of the general $V / R$ 

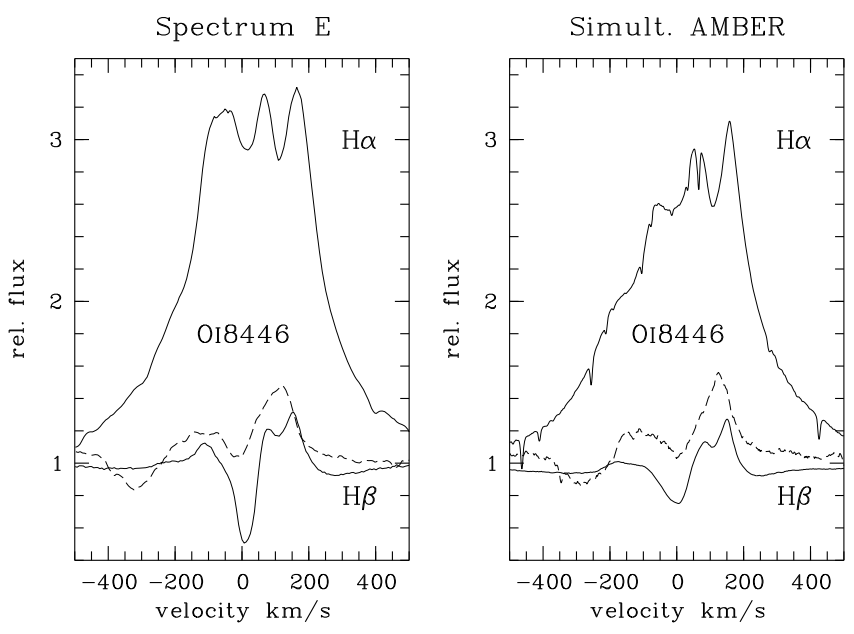

Fig. 4. $\mathrm{H} \alpha, \mathrm{H} \beta$ (solid), and OI 8446 (dashed) line profiles of spectrum "E" (left) and the AMBER-simultaneous FEROS spectrum during triple-peak phases. The absorption on the blue side of O I 8446 is a Paschen line.

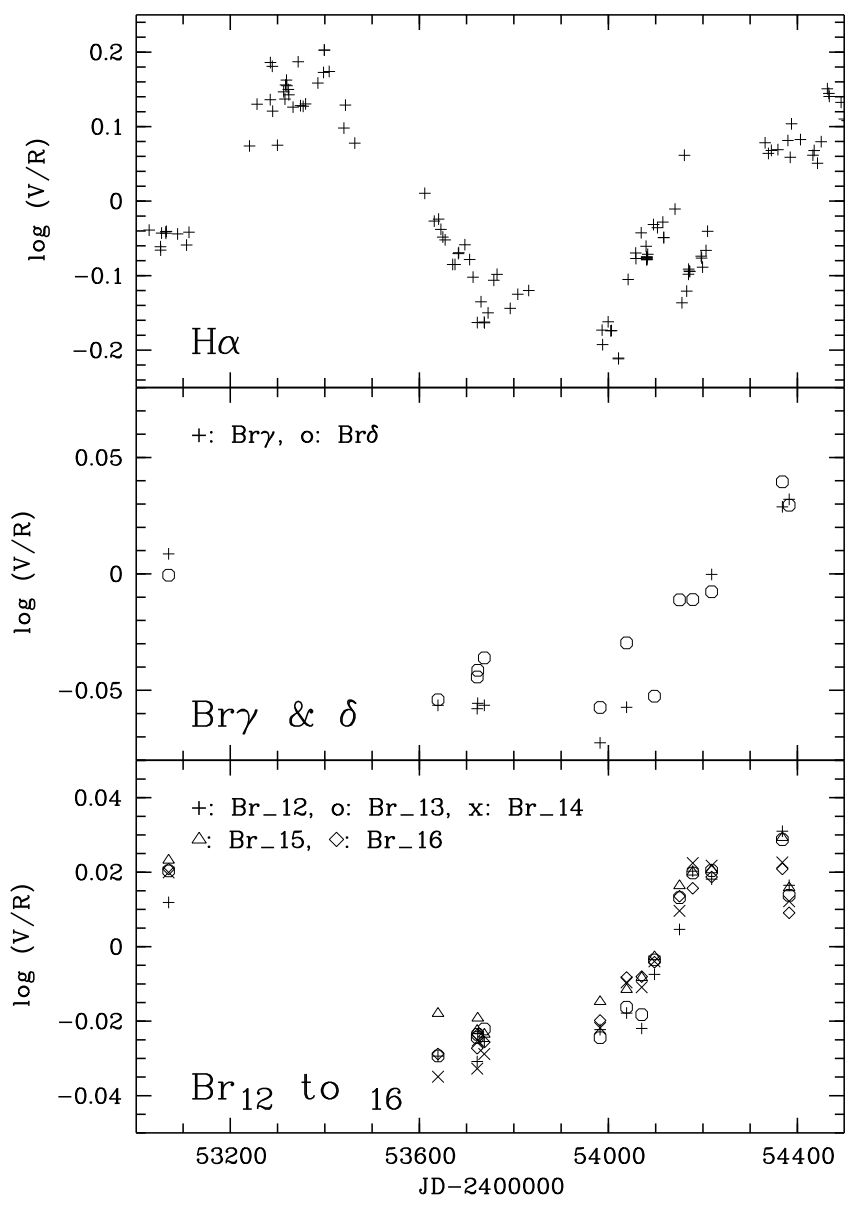

Fig. 5. $V / R$ variations of $\operatorname{Br} \gamma$ and $\delta$ (middle panel) and $\mathrm{Br} 12-16$ (lower panel). $\mathrm{H} \alpha$ values are shown in the upper panel for comparison.

pattern about this phase. The latter includes that, between spectra "E" and "F", BLG shell absorptions shift from zero to moderately positive velocities. Examples are Mg II 4481, Si II 6347, and Fe II 5317 in Fig. 3. This is accompanied by a change in profile symmetry. While in "E" there is a sharp blue edge, "F" exhibits a sharp red edge.

In the NLG shell lines, however, the position of the line minimum as well as the symmetry change in exactly the opposite
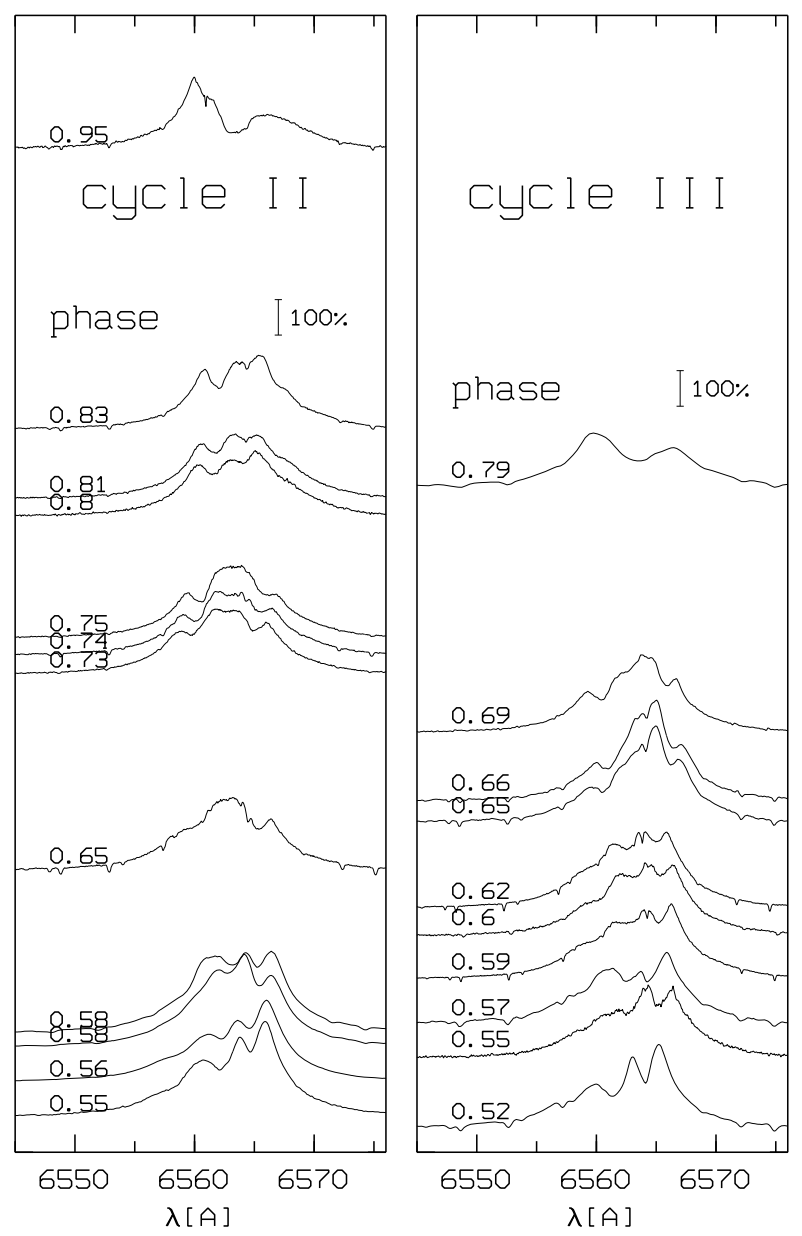

Fig. 6. Evolution of the $\mathrm{H} \alpha$ triple-peak profiles in the two most recent $V / R$ cycles of $\zeta$ Tau. The shifts between the spectra correspond to differences in phase as indicated on the left side. The phase is computed separately for each cycle, using different starting dates ( $V / R$ maxima) and lengths as listed in Table 2. The vertical bar indicates the $100 \%$ continuum level.

Table 4. Optical spectra representative of the $V / R$-cycle. FLASH is the predecessor instrument of HEROS, i.e. the same instrument before the extension with a blue channel.

\begin{tabular}{cccc}
\hline \hline Spectrum & {$[$ JD 24... } & Instrument & Comment \\
\hline $\mathrm{A}$ & 53333.8 & UVES/ESO Archive & nightly average \\
$\mathrm{B}$ & 52009.3 & HEROS/Data Set C & single spectrum \\
$\mathrm{C}$ & 53700.8 & UVES/ESO Archive & night average \\
$\mathrm{D}$ & 52364.3 & HEROS/Data Set C & single spectrum \\
$\mathrm{E}$ & 52725.3 & HEROS/Data Set C & single spectrum \\
$\mathrm{F}$ & 48347.6 & FLASH/LSW Archive & single spectrum \\
\hline
\end{tabular}

way, from low positive velocity to zero velocity in the same two observations. See, e.g., Fe III 5156 and He I 6678 in Fig. 3.

There are very few pure emission lines, e.g. Fe II 6319. But all of them, as well as lines such as $\mathrm{H} \beta$, where emission still dominates over the shell absorption, already show the $V$ peak higher than $R$. That is, the $V / R$ phase of these lines is ahead of the one of $\mathrm{H} \alpha$.

Figure 6 illustrates the evolution of the triple peak profiles in the two most recent $V / R$ cycles. The triple-peak interval in Cycle II lasted for almost 500 days, while in Cycle III it was only 200 days. As can be seen from Figs. 1 and 2, the onset of the triple-peak interval is typically fairly close to phase 0.5 . Much 

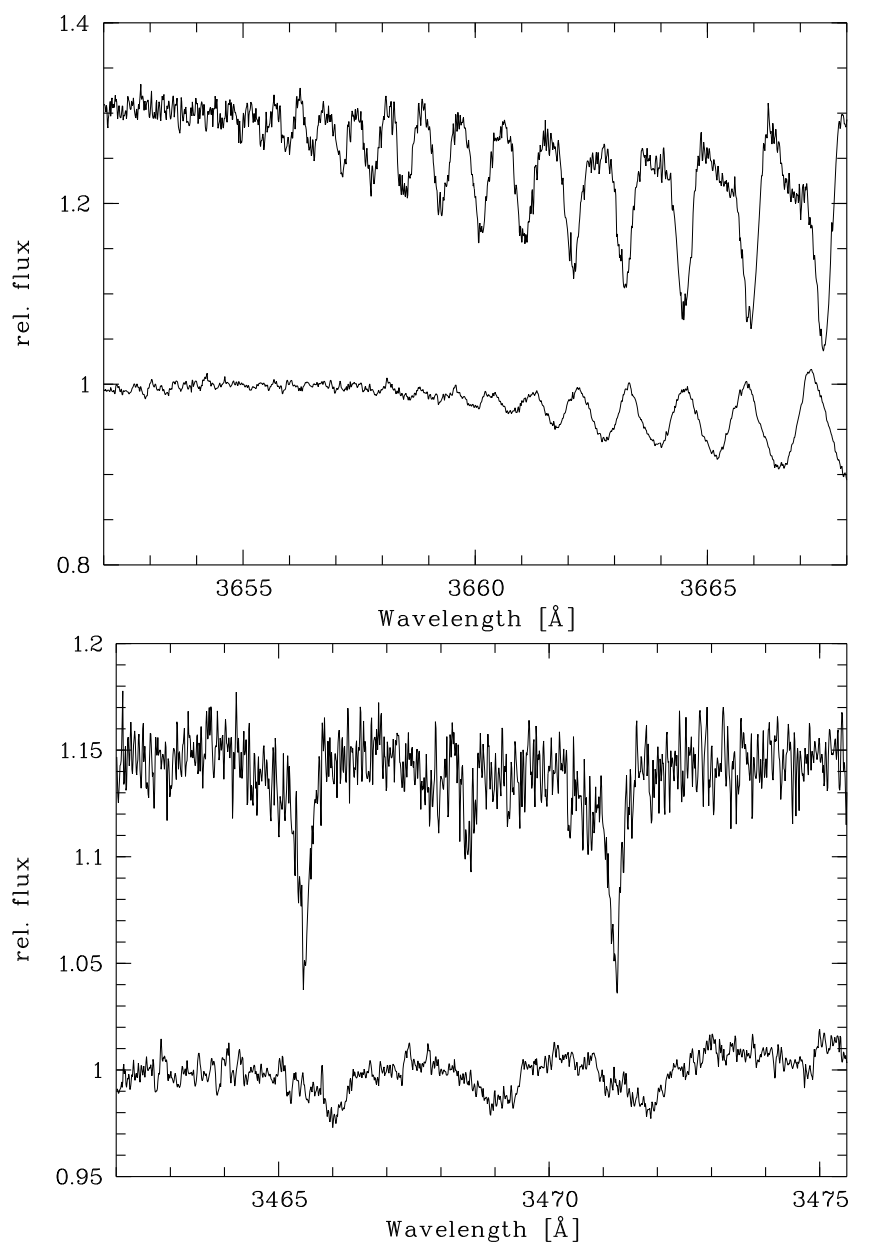

Fig. 7. The region of the Balmer discontinuity (upper panel) and the narrow shell lines (lower panel) in the Balmer continuum in reference spectra "A" (lower plots) and "C" (upper plots), see Sect. 3.5.2.

stronger cycle-to-cycle variations are evident for its terminal phase. In spite of the varying time scale, however, the morphological evolution of the $\mathrm{H} \alpha$ line profile follows approximately the same pattern in all cycles.

\section{Infrared spectral variations}

As for the optical domain, a number of phase-representative reference IR spectra were selected to facilitate the description of the main variability. Their dates are marked as I through $V$ in Fig. 2, and listed in Table 5.

The IR spectra (see Fig. 8) cover the JHK bands and the $\mathrm{H} \alpha$ $V / R$ phases from about 0.3 through 0.8 . Although of lower resolution, some of the conclusions already drawn from the visual spectra are confirmed: the $V=R$ deep absorption phase has the same characteristics in the IR, as far as HI and He I are concerned. In the triple-peak phase, the shell absorption gets shallower in He I and even disappears completely in the higher-order Brackett lines, leaving a pure emission profile although with a pronounced central depression. All observed Brackett lines are double-peaked at all times. But $\operatorname{Br} \gamma$ and $\operatorname{Br} \delta$ develop single-peak profiles when the one in $\mathrm{H} \alpha$ shows a triple-peak structure. Also metallic lines, in particular those of Fe II may show single peak profiles, but at other phases. But it must be suspected that this merger is only apparent due to the limited spectral resolving power.
Table 5. Representative infrared spectra.

\begin{tabular}{ccc}
\hline \hline Spectrum & Date [JD 24... & Comment \\
\hline I & 53722 & 22 days after spectrum "D" \\
II & 53982 & just before triple peak \\
III & 54097 & contemporaneous to AMBER \\
IV & 54281 & just after triple peak \\
V & 54382 & \\
\hline
\end{tabular}

The IR lines provide the basis for a more in-depth discussion of the phase lags between $\mathrm{H} \alpha$ and other lines (Sect. 3.5.4). At some phases, they are well visible even within $\mathrm{H}$ I emission of the Brackett series. In Spectrum III of Fig. 8 the lower Brackett lines still show $V<R$ and, in $\operatorname{Br} \gamma$, even $V \ll R$, but towards the end of the series $V / R$ reaches unity. In a spectrum taken less than a month later, some of the latter lines even appear with $V>R$ while $\operatorname{Br} \gamma$ looks unchanged. The $V=R$ state is also reached in $\mathrm{He}$ I, Fe II, and $\mathrm{Mg}$ II. These two particular observations (incl. Spectrum III) were obtained almost simultaneously with the AMBER data described below.

The phase lag between higher- and lower-order Brackett lines is also well visible in Fig. 5. Already the first IR observation on JD 2453069 shows that, while $\log (V / R)$ is still below unity for $\mathrm{H} \alpha$, it is about unity for $\operatorname{Br} \gamma$ and $\mathrm{Br} \delta$, and still larger for higher Brackett lines. In general, the $V / R$ values for both $\mathrm{Br} \gamma$ and $\operatorname{Br} \delta$ precede the $V / R$ curve of $\mathrm{H} \alpha$, but clearly lag behind the ones of the $\mathrm{Br} 12-16$ lines. The $V / R$ amplitude of the $\mathrm{Br} 12-16$ lines is slightly lower than the ones of $\operatorname{Br} \gamma$ and $\operatorname{Br} \delta$. But it is lower by a factor 8-10 relative to the one of $\mathrm{H} \alpha$.

Contrary to $\mathrm{H} \alpha$ (see Fig. 2), a distortion of the $V / R$ curve around phase 0.6 due to triple-peak profiles cannot be recognized in any IR lines. Observations at higher spectral resolution are desirable to establish this definitively.

A very conspicuous type of behavior without counterpart in the visible range is associated with the infrared $\mathrm{CI}_{\mathrm{I}}$ lines (see upper right panel of Fig. 8). IR multiplets 1 and 24 of this species are seen in emission in six out of fourteen IR spectra (JD24: $53069,53639,53722,53723,54038$, and 54 070) and absent in the others. Since intermediate cases were not observed, this may constitute some type of "on/off" behavior. Contrary to the typical circumstellar lines described above, these lines have a symmetric single-peak profile and exhibit no variability other than their being either present or absent. No relation could be found of the presence or absence of the C I emission to the $V / R$ cycle or the 132.9735-d orbital period. It was also checked that the variations cannot be produced by an overlapping $\mathrm{OH}$ telluric emission (Rousselot et al. 2000).

\section{Interferometric results}

\subsection{Results from $A M B E R$}

In Fig. 10, the left panel shows the coverage of the $u v$ plane by our AMBER observations. Following the ESO/VLTI documentation, the following baseline position angles were accepted: $U 1-U 3=32.369, U 1-U 4=60.396$ and $U 3-U 4=$ $110.803 \mathrm{deg}$. The right panel shows the absolutely calibrated squared visibilities in continuum. Because the transfer function is very uncertain, the final precision is rather poor, errors are as high as $20-30 \%$. The model visibilities for baselines along the major and minor axis of the $\zeta$ Tau model derived from the CHARA $K$ '-band observations by Gies et al. (2007) are 

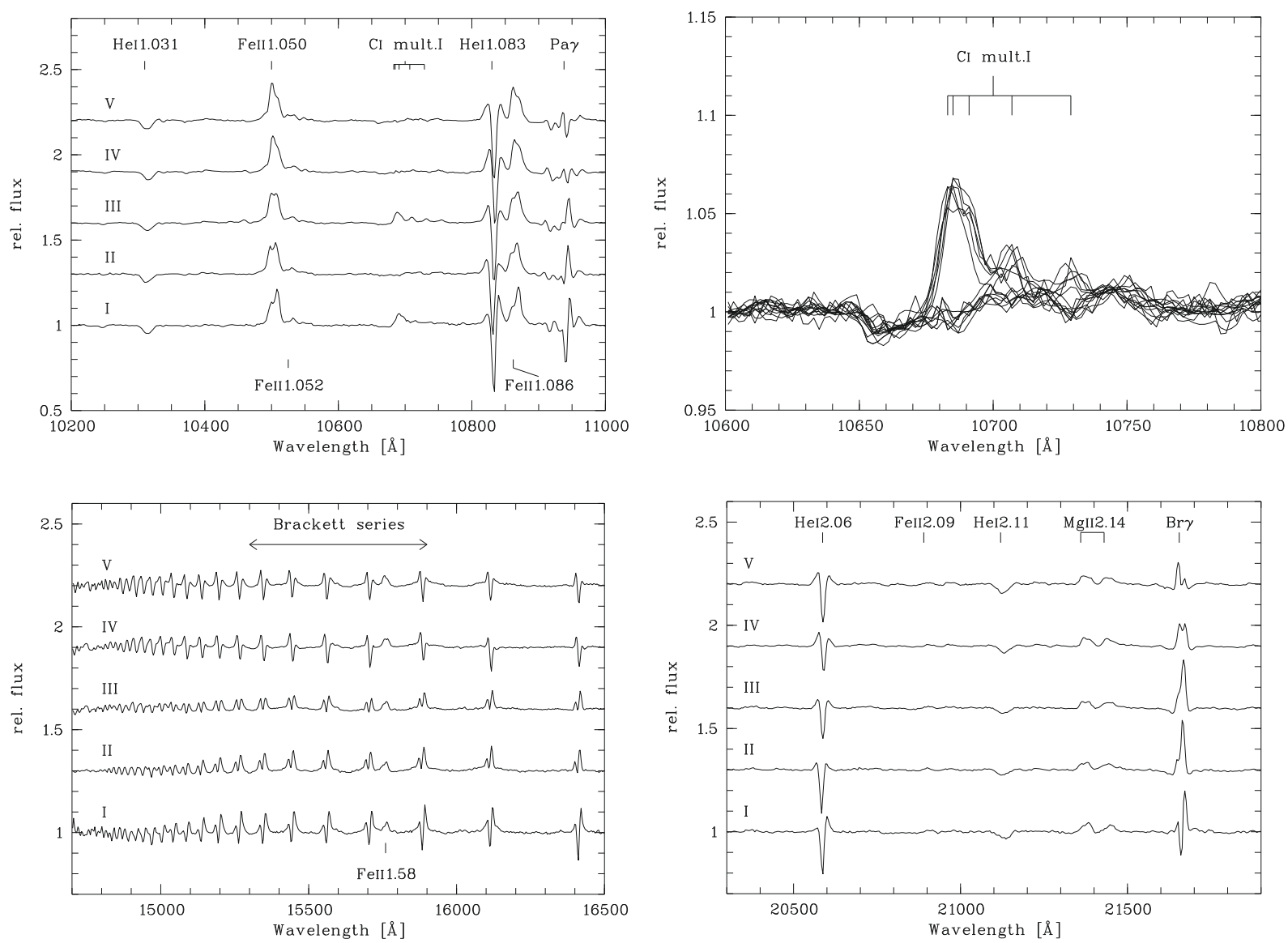

Fig. 8. Selected spectral windows in the $J, H$, and $K$ bands. Observing dates of spectra $I$ through $V$ are given in Table 5; their phases in the mean $\mathrm{H} \alpha$ cycle are marked in Fig. 2. Pa $\gamma$ is blended with He I 16600 and two magnesium lines. Spectrum III is the one closest in time to the AMBER observations. The upper right panel combines all spectra of C I multiplet 1 in the $J$-band, illustrating its on/off behavior.

over- plotted. The AMBER continuum observations - in spite of the large scatter - are compatible with the CHARA model. However their accuracy and the fact that $\zeta$ Tau is only marginally resolved by AMBER/VLTI in the $K$-band continuum prevent us to test the model in more detail.

AMBER spectro-interferometric measurements were combined with the CHARA model in order to derive visibilities and phases in $\mathrm{Br} \gamma$ and $\mathrm{He}$ I $2.06 \mu$ emission lines (see Sect. 2.3). The differential values are shown in Figs. 11 and 12 for the regions around $\mathrm{Br} \gamma$ and $\mathrm{He} \mathrm{I} 2.06 \mu$, respectively. In all baselines, the signal is much stronger in the $\operatorname{Br} \gamma$ line $(2.17 \mu \mathrm{m})$, but it is also clearly detected in the He I line $(2.06 \mu \mathrm{m})$. The drops in visibility prove that the $\mathrm{Br} \gamma$ and $\mathrm{He} \mathrm{I}$ emitting regions are more extended than the continuum emitting region. Moreover, an asymmetry in the visibility profile is clearly seen, with the visibility in the red part being lower than in the blue part. Fitting these visibility profiles across the lines requires proper modeling. Such a work is presented in Paper II.

Apart from the visibility signature, also a phase effect was found in all baselines for $\operatorname{Br} \gamma$, indicating that the photocenter across the line is shifted with respect to the photocenter in the continuum. To allow a geometrical representation of the AMBER phases, the differential-phases $\phi(\lambda)$ across the $\operatorname{Br} \gamma$ line were converted into $2 \mathrm{D}$ astrometric shifts $p(\lambda)$ by inverting the well-known formula for marginally resolved interferometric observations (Lachaume 2003):

$\phi(\lambda)=-2 \pi \cdot \boldsymbol{p}(\lambda) \cdot \frac{\boldsymbol{b}}{\lambda}$ where $\boldsymbol{b}$ is the interferometric baseline vector projected onto the sky. Uncertainties were propagated to the astrometric vector $\boldsymbol{p}$ by standard formulas. Neither the uncertainty in the baseline length nor in the spectral calibration were taken into account, because they affect all spectral bins in the same way.

Figures 11 and 12 prove the presence of phase differences of the $V$ and $R$ emission components with respect to the continuum. The observed (differential) phases are the flux weighted vector sum of the source phases of the continuum and line components. With a physical model of the disk and radiative transfer, the fluxes are known and the phase differences between the continuum and line emitting regions can be translated into their angular separation. Such a model is presented in Paper II. The model-independent conclusion is that the line emitting regions and the continuum source lie in one common plane but that the $V$ and the $R$ components arise from different locations that do not coincide with the continuum source.

Figure 13, left panel shows the computed relative offsets in angular units. The phases across the $\operatorname{Br} \gamma$ line is converted into 2D photocenter shift in the plane of the sky. The photocenter is displaced toward the NW direction in the blue part of the line, and toward the SE direction in the red part of the line. This is the clear signature of the rotating disk emitting the $\mathrm{Br} \gamma$ line. The position angle of the displacement is perfectly compatible with the position angle of the CHARA model based on measurements made in the $K^{\prime}$ continuum, as shown in the right panel of Fig. 13. Moreover, the amplitude of the photocenter displacement is significantly larger in the red wing $(280 \mu \mathrm{as}, \mathrm{SE})$ than in the blue wing $(120 \mu \mathrm{as}, \mathrm{NW})$. The simplest explanation is an asymmetry 

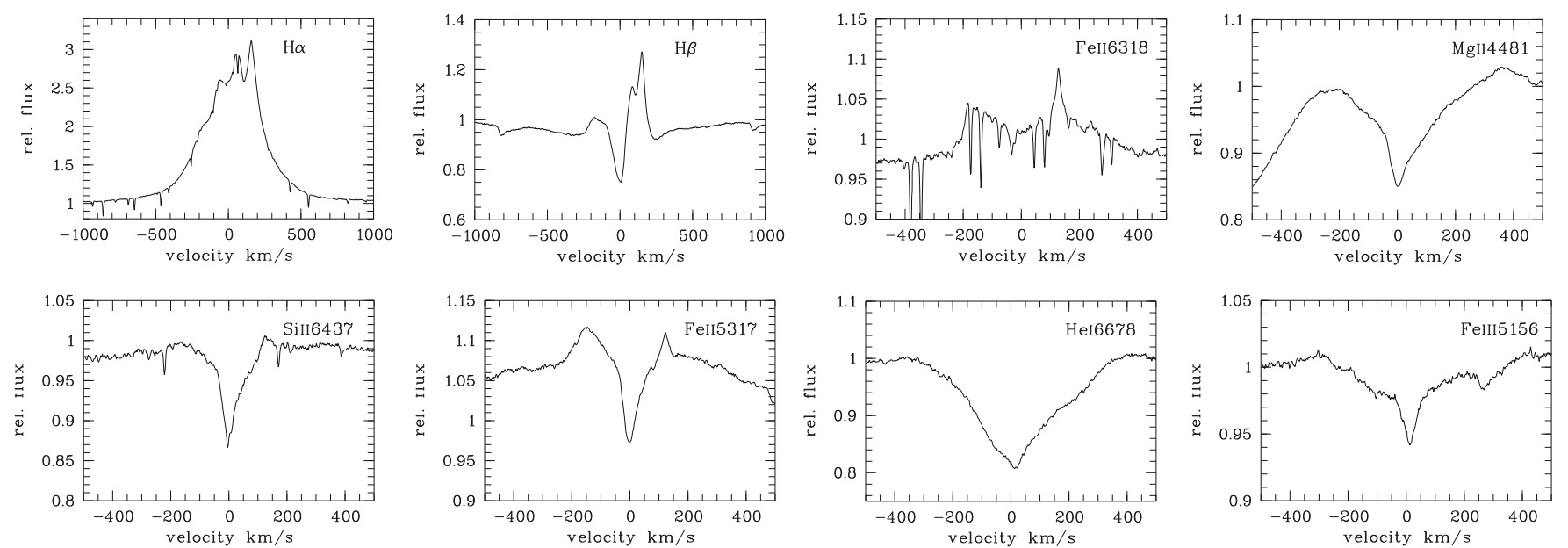

Fig. 9. Visual spectroscopic state of $\zeta$ Tau during the AMBER observations.
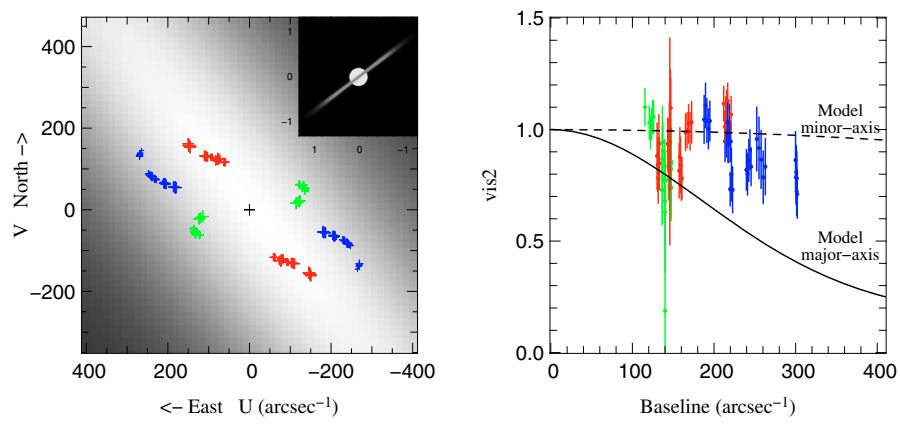

Fig. 10. Schematic comparison of the VLTI/AMBER observations with the CHARA model by Gies et al. (2007). In the colour plot available in the on-line version of the paper, the green, red and blue colours correspond to the UT 3-4. UT 1-3 and UT 1-4 baselines, respectively. Left panel: Coverage of the $u v$ plane by AMBER observations of Dec. 12, 2006. The sub-frame in the upper right corner shows the CHARA model projected on the sky (before the transformation). The sub-frame covers the region of about 3 mas x 3 mas and corresponds to the $K$-band continuum. The squired visibility of the AMBER observations is linearly proportional to the size of symbols, for the CHARA model (in the sub-frame and transformed in the background) to the brightness. Right panel: Absolutely calibrated continuum squared visibilities as a function of the baseline length. Squared visibilities of the major and minor disk axes derived from the CHARA model are overplotted.

in the $\mathrm{Br} \gamma$-emitting material, with the SE part being brighter and/or more extended than the NW part. Again, modeling of this signal in the framework of the one-armed disk-oscillation model is developed in Paper II.

\subsection{Optical and IR spectra accompanying the AMBER observations}

When AMBER observed $\zeta$ Tau, simultaneous FEROS optical spectra as well as quasi-simultaneous IRTF spectroscopy were also obtained. Figure 9 depicts representative visual line profiles. Further FEROS observations were made throughout December 2006, which differ but little from the one at the time of the VLTI observations. In the IR, Spectrum III (see Fig. 8) comes closest (12 days before) in time to the AMBER observations. A next spectrum, obtained 16 days after AMBER data, is very similar. Therefore, the low variability in both optical and IR spectra suggests that the selected spectra represent well the dynamics of the disk at the time of the AMBER observations.
Table 6. Mean polarimetric properties, taking into account only HPOL data after JD 2490000.

\begin{tabular}{ccccc}
\hline \hline $\begin{array}{c}\text { Spectral } \\
\text { Band }\end{array}$ & \multicolumn{2}{c}{ Pol. degree } & \multicolumn{2}{c}{ Pol. angle } \\
Mean [\%] & $\sigma$ & Mean [deg] & $\sigma$ \\
\hline$U$ & 0.99 & 0.14 & 32.3 & 3.5 \\
$B$ & 1.59 & 0.08 & 31.6 & 1.6 \\
$V$ & 1.46 & 0.08 & 31.3 & 1.5 \\
$R$ & 1.30 & 0.06 & 31.3 & 1.2 \\
$I$ & 1.18 & 0.06 & 31.5 & 1.5 \\
\hline
\end{tabular}

As IR spectrum III shows (Fig. 8), the emission is almost symmetrical in He I $2.06 \mu \mathrm{m}$, so that the much lower amplitude of the phase signature in the same line is in good agreement with the spectroscopic data. The distribution of the He I $2.06 \mu \mathrm{m}$ emitting material is symmetric with respect to both systemic velocity and location of the continuum source.

The situation is different for $\mathrm{Br} \gamma$ but the reasoning is the same: the closure phase is undoubtedly non-zero for this line and proves a spatial asymmetry in the brightness distribution of the system (continuum vs. line-emitting region). At the same time, the $V / R$ value of $\mathrm{Br} \gamma$ is far from unity. The combination of these two asymmetries places the bulk of the $\mathrm{Br} \gamma$-emitting region to the receding sector of the disk.

The scheduling of the AMBER observations was very successful in that they could be made during a triple-peak phase of the $\mathrm{H} \alpha$ emission. However, with single-epoch data it is difficult to say whether they harbor any related hidden anomaly. For a discussion of the azimuth (in a not point-symmetric model) of the region of formation of this spectral structure see Sect. 7.6.

\section{Polarimetric results}

Since the $V / R$ cyclicity before JD 2490000 is not well constrained by the observations and, as described in Sect. 3.2, $\zeta$ Tau reached a stable disk state only after that date, only polarization measurements obtained after JD 2490000 were taken into account for the computation of the mean values shown in Table 6.

The polarization angle (PA) is the same in all bands within both the individual uncertainty and the standard deviation. Although the standard deviation of the PA measurements is slightly higher than the uncertainty estimated for a single measurement, this difference is not large enough to conclude a 

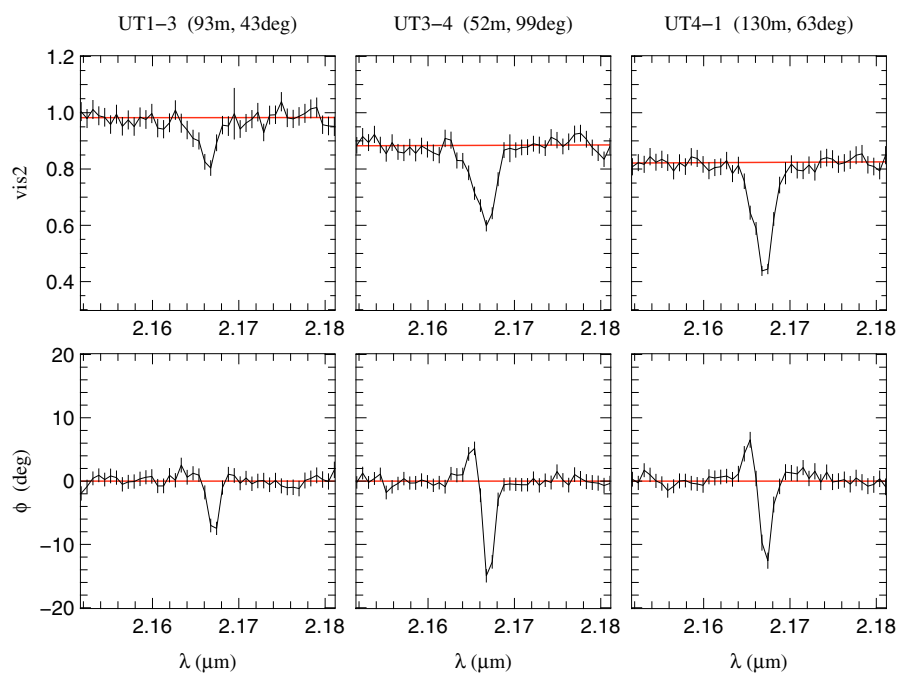

Fig. 11. AMBER visibilities and phases around $2.18 \mu \mathrm{m}$ normalized to the model of Gies et al. (2007).

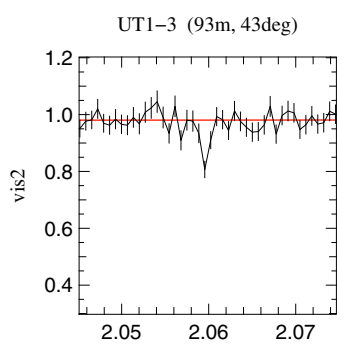

UT3-4 $(52 \mathrm{~m}, 99 \mathrm{deg})$

UT4-1 (130m, 63deg)
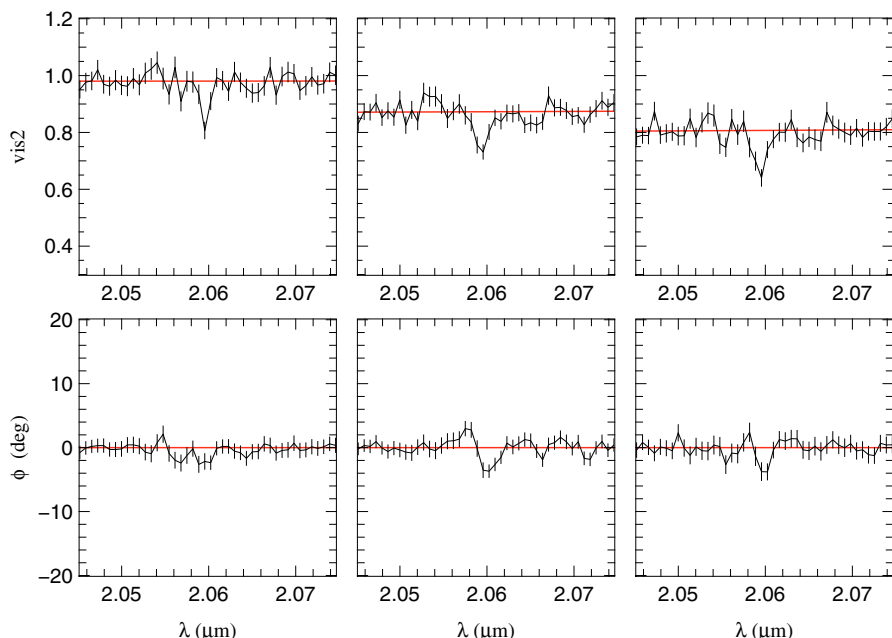

Fig. 12. Same as Fig. 11 but around $2.06 \mu \mathrm{m}$. The signal is significantly weaker but the He I line is still detected.

variable polarization angle. Even after inclusion of all data, i.e. also the observations taken before the $V / R$ cycle is well defined, the values for the PA in Table 6 would change only in the last digit, if at all, so that the conclusion about a non-changing angle is valid over the entire period of observation.

Other than the angle, however, the polarization degree does vary. The variability shown in Fig. 14 can be disentangled into two components. A slow and secular change is visible well in the VRI and, though somewhat noisy, also in the $U B$ bands in Fig. 14. This behaviour is in agreement with the general evolution of the $\mathrm{H} \alpha$ disk as described in Sect. 3.2: the mean polarization degree is lower before JD 2448000 , then rises until JD 2450000 , and after that remains stable again. Superimposed on that pattern is short-term variability. Unfortunately, the data do not cover the timescales required to properly constrain the short-term component. However, the observed behavior is not in contradiction with polarimetric variability produced by a series of discrete and individually rather minor ejections of matter into the immediate stellar environment, as already suggested in Sect. 3.1.
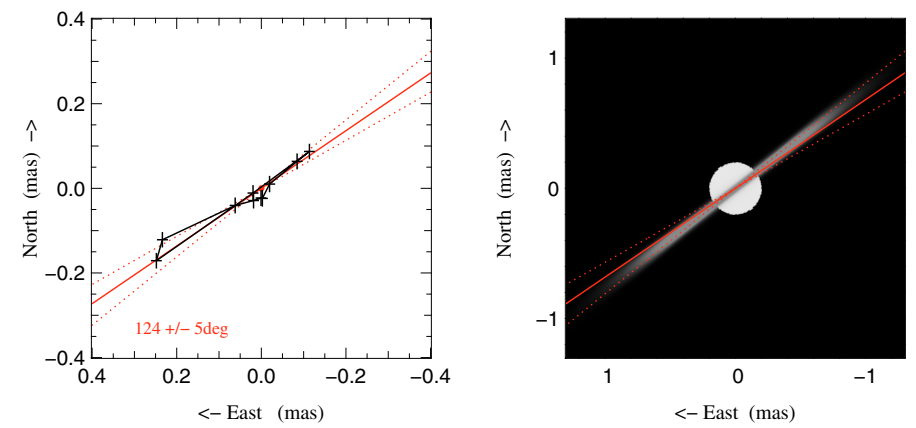

Fig. 13. Left: photocenter shifts derived from the AMBER relative phases across $\mathrm{Br} \gamma$. The maximum shift is about 0.4 mas within the plane of the circumstellar disk, while no significant offset perpendicular to it can be found (black line). Right: the position angle derived from our differential data overplotted on the model of Gies et al. (2007).

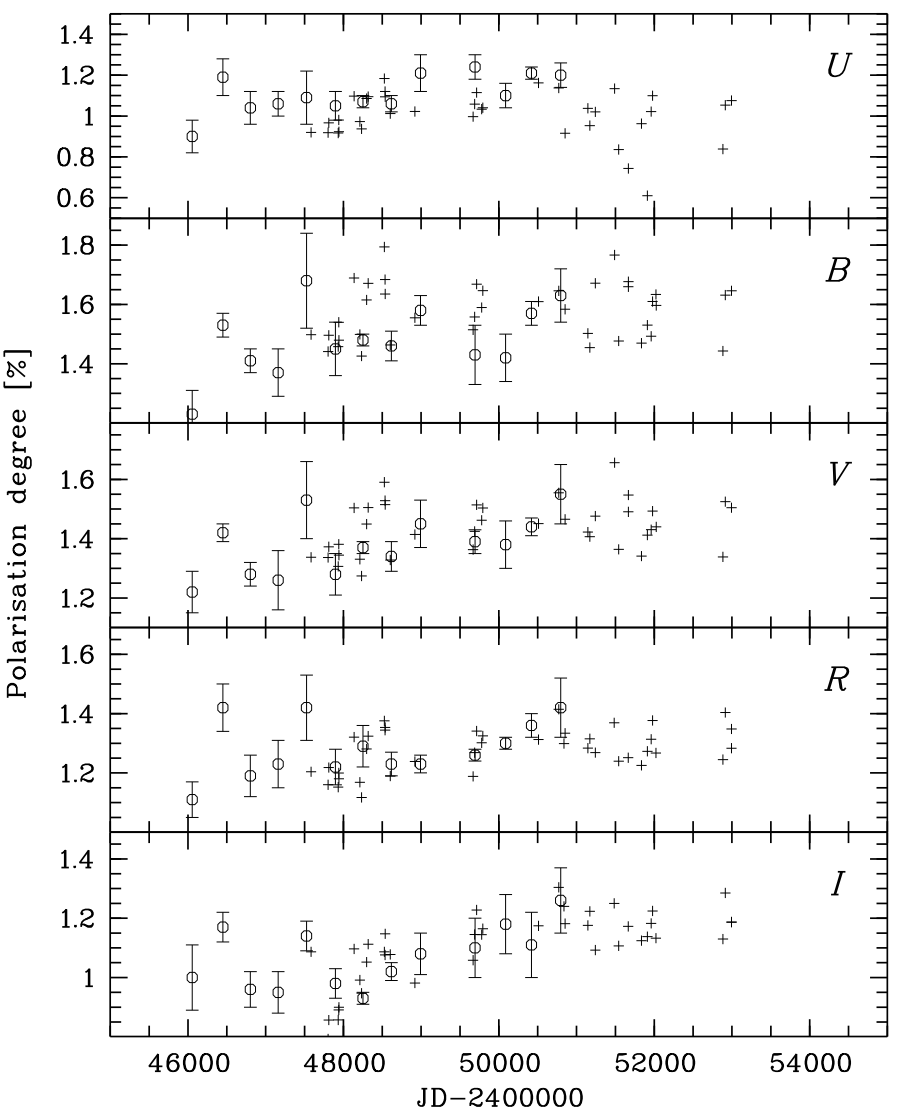

Fig. 14. Temporal behaviour of the polarization degree. Data from McDavid (1999) are shown as circles. The uncertainty of the HPOL data (+) is about the size of the symbols, i.e. $0.01 \%$.

In summary, neither the variability of the polarization degree nor the polarization angle, which is stable, can be connected to the cyclic $V / \mathrm{R}$ variability. No modulation due to the orbital period could be detected.

\section{Synopsis and discussion}

\section{1. $\zeta$ Tau spectral type}

Table 3 lists several near-UV He I lines as having purely photospheric profiles. They offer the opportunity to shed some light on the various discrepant spectral types that have been published for $\zeta$ Tau. A comparison of the high-quality UVES data to several early B-type stars in the UVES POP-library (Bagnulo et al. 2003) showed unambiguously that the observed strength of these 
lines is inconsistent with an effective temperature corresponding to a spectral type of $\zeta$ Tau later than B2. However, an exact redetermination of the spectral type is beyond the scope of this study.

\subsection{Binarity}

With $\zeta$ Tau having an unseen companion, any variability must be checked for a relation to the $132.9735 \mathrm{~d}$ orbital period established by Harmanec (1984). However, the strength of the disk during the observations implies that the vast majority of the spectral lines are contaminated by line emission primarily varying on a different time scale. The other lines are mostly too shallow for radial-velocity measurements that would permit a verification of Harmanec's amplitude of $K \approx 10 \mathrm{~km} \mathrm{~s}^{-1}$ to be attempted, which was derived from observations at more $V / R$-quiescent epochs. The photospheric He I lines are all in the blue spectral region, which is covered only by a small number of spectra used in this study.

It follows that the used dataset of optical spectra, focused on the $\mathrm{H} \alpha \mathrm{V} / \mathrm{R}$ variations is not suitable for a refinement of orbital parameters. As a compromise only a check of the period using the HeI 4026 line was performed. Radial velocities were measured by fitting Gaussians to the line core. Phasing this data with Harmanec's ephemeris gives a plot similar to his Fig. 1a but with higher errors and a few outliers. A time-series analysis finds the second strongest peak corresponding to a period of $131.6 \pm 0.5 \mathrm{~d}$, in reasonable agreement with Harmanec. The strongest peak present is a nearby alias of this one, probably enhanced by noise effects. No other quantity investigated in this work shows a significant modulation with the orbital period.

Even over the given large spectral range, lines due to a secondary, either hot or cool, were not found. In particular, no trace of He II 4686 was detected that would have indicated a hot subdwarf-like companion.

Re-analysis of the publicly available photometric data (Guo et al. 1995; Pavlovski et al. 1997; Harmanec et al. 1997, and data from the HIPPARCOS-mission) did not confirm the presence of photometric eclipses suggested by Božić \& Pavlovski (1988). Rather, the fainting at phase zero they observed seems to be an event unrelated to the orbital phase. There are more such fadings but at random phases and none of them at phase zero.

\subsection{Disk orientation on the sky}

In an axi-symmetric circumstellar disk the PA is in general perpendicular to the disk plane $^{3}$ and thus parallel to the disk position angle $(\chi)$.

The spectropolarimetric monitoring of $\zeta$ Tau from the PBO observatory shows that the polarization angle has been remarkably constant from at least 1989 to 2004 (see Sect. 6). The average $V$-band polarization angle is $\mathrm{PA}_{\mathrm{PBO}}=31.3 \pm 1.5^{\circ}$ with an individual measurement uncertainty of $1 \mathrm{deg}$. The data from Limber Observatory, taken between 1984 and 1997 (McDavid 1999), gave very similar results: $\mathrm{PA}_{\text {Limber }}=32.5 \pm 1.1^{\circ}$, and a typical individual uncertainty of $0.5^{\circ}$.

Further, there are three independent measurements of the position angle from interferometric studies. Quirrenbach et al. (1997) determined from a 2D Gaussian fit for data from the Mark III interferometer a value of $\chi_{\text {MarkIII }}=31 \pm 4^{\circ}$. Using data from NPOI, Tycner et al. (2004) obtained $\chi_{\mathrm{NPOI}}=28 \pm 4^{\circ}$, while

\footnotetext{
${ }^{3}$ For very optically thick disks, the polarization can be parallel to the disk plane.
}

Gies et al. (2007) report $\chi_{\mathrm{CHARA}}=37 \pm 2^{\circ}$ from their CHARA data. Finally, the VLTI data of Sect. 5 show that $\chi_{\text {AMBER }}=$ $32 \pm 5^{\circ}$.

The orientation of the disk on the sky, as indicated by all these measurements, has not changed since 1984, and in particular there seems to be no binary phase-locked variability throughout the $V / R$ cycle, at least within the individual measurement uncertainties. The stability of the disk position angle is typical for Be stars, the only few counter-examples ever found are suspected to undergo precession of a non-equatorial disk (Hummel 1998; Hirata 2007).

\subsection{V/R cycle length}

The analysis of the up to now most comprehensive observational data set of $\zeta$ Tau confirmed that its $V / R$ variations follow a cycle with relatively stable amplitude and length of 1405-1430 days duration during the present $V / R$ variable phase starting at the beginning of nineties. The length is different and much more stable than during the previous $V / R$ active phase in 1955-1980.

Table 2 shows that the cycle length varies and suggests a correlation between the cycle length and the duration of the triplepeak epoch. In fact, the differences in cycle length seem to be dominated by the duration of the triple-peak phase. For instance, the lengths of Cycles II and III differ by 291 days. But after subtraction of the duration of the triple-peak epochs, the remainders of the cycles are of the same length (1033 and 1027 days, respectively). The lack of an explanation of the variation of the duration of triple-peak phases may limit models for the basic $V / R$ activity as well. Fortunately, at less than $\pm 10 \%$, the quantitative effect is small.

The well-observed $V / R$ maxima in $\mathrm{H} \alpha$ have been decreasing through Cycles I to III. This may be indicative of a decrease also of the amplitude of the perturbation causing the $V / R$ variations.

\subsection{V/R phase differences between emission lines}

The relative shifts in the $V / R$ variations of Balmer, Brackett, He I, and metal emission lines should probe the disk at decreasing distances from the central star and so place considerable constraints on any explanation of the cyclic $V / R$ variability of $\zeta$ Tau (and other Be stars). The phase lag between $\mathrm{H} \alpha$ and the higher Brackett lines is of the order of $\Delta \phi \approx 0.25$ (see Fig. 5). Unfortunately, any quantitative modeling is somewhat hampered by the medium spectral resolution of both the interferometry and IR-spectroscopy.

From a subset of the present IR spectra, Wisniewski et al. (2007) derived the opposite $V / R$ phase relation between IR lines and $\mathrm{H} \alpha$. The larger phase and wavelength coverage of the present study appears to exclude this possibility.

\subsection{Triple-peak $\mathrm{H} \alpha$ profiles}

The present compilation of spectra maps the evolution of the triple-peak profiles in Cycles II and III in much detail. Qualitatively, it follows a very similar pattern in either cycle. But the duration of the triple-peak phases is variable and may last from 200 to 500 days. They are not phase-locked to the companion star. However, this does not invalidate the small-number statistics produced by Rivinius et al. (2006), who report that triple-peak line profiles only occur in shell stars that are both $V / R$ variable and multiple.

As is apparent from Fig. 3, the triple-peak profile in $\mathrm{H} \alpha$ is accompanied by a somewhat disturbed profile in $\mathrm{H} \beta$. But other 
non-hydrogen emission lines in the visual range are largely unaffected. Although the lower resolution of the IR spectra leaves some space for undetected line profile deformations, IR emission lines, too, (including those of $\mathrm{HI}$ ) do not seem to undergo related variations.

The absence of triple peaks from optically thin emission lines and in particular the stability of O I 8446, which probes the $\mathrm{H} \alpha$-forming region due to $\mathrm{Ly} \beta$ resonance pumping, suggest that the triple-peak feature does not probably correspond to an actual density structure. Triple peak profiles have so far only been reported in shell stars. This detail, too, would not easily be explained by real density enhancements.

This gives rise to the speculative conjecture about a change in the local escape probability due to distortions of the local velocity field, which of course would have an effect only on optically thick lines, like $\mathrm{H} \alpha$. Another possibility, guided by the finding by Štefl et al. (2007) that triple-peak emission lines occur in binary Be stars, might be some resonance between the orbital motion of the companion stars and the orbital motion of gas in the outer disk.

$\mathrm{H} \alpha$ and $\mathrm{H} \beta$ are not the only optically thick emission lines, though: It is commonly accepted that the Paschen and Brackett lines as well as some strong IR Fe II emission lines form closer to the star than the first few lines of the Balmer series. Since only $\mathrm{H} \alpha$ and $\mathrm{H} \beta$ show some form of triple peak, this may point at the outer regions of the disks as the locus of the physical variability. Such a region was on the line of sight at the time of the AMBER observations. At that time, the bisector line of the $\mathrm{H} \alpha$ emitting region was close to the line of sight, whereas the bisector lines of the regions of formation of emission lines requiring higher proximity to the exciting central star passed the line of sight up to one year earlier.

\subsection{Narrow- and broad line shell absorptions}

As described in Sect. 3.3, NLG lines are formed in a broad diversity of ionic species and over a wide range of ionization and/or excitation potentials beginning with $\mathrm{O} I$ and $\mathrm{Na} I$ and extending to Fe III. Conversely, BLG characteristics are exclusively associated with transitions expected from comparatively cool conditions; examples are $\mathrm{Mg}$ I or O I. The simultaneous, let alone long-term, presence of both Fe III and Mg I shell absorption is very unusual. A search of the large FLASH, HEROS, and FEROS database of Be spectra (Rivinius et al. 2003) furnished no second example.

The occurrence of lines with persistent mixed BLG and NLG characteristics suggests that the circumstellar disk does not have a simple and smoothly varying structure. Rather, NLG and BLG components seem to form in two spatially separated regions with distinct physical conditions. Simple common-sense considerations lead to conflicting conclusions about the location of these regions relative to the central star.

On the one hand, their higher excitation would place the NLG closer to the star and the lower-excitation BLG in the outer disk. On the other hand, the line profiles point to the opposite, provided that the disk dynamics is crudely Keplerian: The narrowness of the NLG, which in some UV lines hardly exceeds the thermal width, as well as their lower RV amplitudes suggest, then, that the region of formation of NLG lines is relatively far from the central star. The broader BLG lines would form in the inner disk, where the velocity ranges are larger.

Apart from their width, NLG and BLG lines also differ in the variation of their equivalent width with $V / R$ phase. While BLG lines are strongest in the second half of the cycle, NLG lines are strongest around phase 0.25 .

The radial-velocity variations of both groups have comparable amplitude through the $V / R$-cycle (see Fig. 3). However, there is a phase difference of about $\Delta \phi \approx 0.25$ with the NLG trailing the BLG.

\section{Conclusions}

$\zeta$ Tau is a Be-shell star. Its spectral type is B2 or earlier one. Both interferometry and polarimetry demonstrate that its circumstellar disk is flat and seen edge-on. The assumption that all Beshell stars are observed edge- and equator-on has been vital for the analysis by Rivinius et al. (2006) of the fractional critical rotation rates of Be stars.

The plane of the disk has remained stable for decades. Warping or tilting, as diagnosed in other Be stars by Hummel (1998), may be present but was not detected. The disk seems to have been persistent for about a century. But the strength of emission and shell absorption lines has varied on time scales of years to decades.

The photocenter of the $\operatorname{Br} \gamma$ line emission lies in the plane of the disk but is offset from the continuum source. The same could not be diagnosed from the weaker He I $2.06 \mu \mathrm{m}$ emission line. This may be a data-quality issue or result from an alignment, at the time of the observations, of the photocenter of the He I line with the continuum source.

The Br $\gamma$ result may also resolve a general ambiguity in the interpretation of $V / R$-asymmetric emission lines. From the spectra alone one cannot decide whether the $V / R$ asymmetry is only in velocity or also in configuration space. The VLTI observations show that the latter is true in $\zeta$ Tau.

Like some other, but by far not all, Be stars, $\zeta$ Tau is a binary. The companion remains undetected at optical and IR wavelengths. An intensive search in a dense series of multiwavelength observations of a very broad range of electromagnetic observables did not furnish any effect of the companion on the long-term variability or orientation of the disk. The variability of the circumstellar disk can, therefore, be considered intrinsic to the disk itself and the central B-type star.

The most prominent spectroscopic signature of the disk activity is the cyclic $V / R$ variability. Its amplitude can for decades drop below the level of easy detectability. Pronounced $V / R$ variations were resumed about 1992 . Since 1996 , three complete $V / R$ cycles were observed. Their similarity in length and amplitude has enabled a detailed phenomenological description of the variability.

The formal mean cycle length is 1405-1430 days, with cycle-to-cycle variations of less than $\pm 10 \%$. The basic $\log (V / R)$ curve for $\mathrm{H} \alpha$ is smooth and symmetric. However, about the $V / R$ minimum a perturbation develops, which has been qualitatively the same in all three of the most recent cycles. The $\mathrm{H} \alpha$ emission profile develops a comb-like structure at its top, which consists of three small peaks separated by equally small depressions. The duration of this phase varies from cycle to cycle, and the changes may be the main reason of the variation in length of the main cycle. In the available observations, triple-peak profiles are restricted to $\mathrm{H} \alpha$, with traces showing up in $\mathrm{H} \beta$. Therefore, the underlying perturbation may be confined to the outer disk.

All emission lines partake in the $V / R$ variability. But there are line-specific shifts in phase of up to $25 \%$ of the cycle length. $\mathrm{H} \alpha V / R$ lags behind $\mathrm{Br} \gamma$, which trails the higher Brackett lines. Metallic lines typically behave like the higher Brackett lines. This supports the notion that the phase lag increases with decreasing density and increasing distance from the central star. 
The shell absorption lines crudely fall into two different groups mainly distinguished by the line widths. Low-ionization species and low-excitation lines are broader than the ones involving higher energies. Some lines also share the behavior of both groups so that each group may predominantly arise from different locations. If the disk motions are basically Keplerian and density and temperature mainly drop with distance from the central star, the grouping provides seemingly contradictory diagnostics of these locations relative to the central star. Both groups undergo similar radial-velocity variations but the broad lines lead in phase by about one-quarter of a $V / R$ cycle.

The only variability apparently unrelated to both the $V / R$ cycle and the binarity is the occasional presence of infrared $\mathrm{CI}$ emission.

Discrete major mass loss events were not observed. But observations of other Be stars and the relative constancy of the total emission strength suggest that minor ones have likely taken place. They may provide a partial explanation of not fully repetitive variations.

The complexity of the observations and especially the various cyclically repeating phase differences show that the underlying perturbations are not both point-symmetric and radially monotonic. Consequently, the perturbations are hardly reconcilable with roughly spherically symmetric models as, e.g., the one of Doazan \& Thomas (1987). Major outbursts might temporarily lead to such a situation but the cyclic variability would probably require them, too, to be cyclic. A perturbation that propagates in a spiral-like pattern has a physical foundation in global $m=1$ disk oscillations, which will be developed into a fully self-consistent model in Paper II (Carciofi et al. 2009).

Acknowledgements. The polarimetric observations were supported in part by NASA via contract NAS5-26777 with the University of Wisconsin and grants NAG5-3447 and NAG5-8054 to the University of Toledo. We thank Ken Nordsieck for access to HPOL. We also thank the many members of the PBO observing and data reduction teams over the years, with special thanks to Marilyn Meade and Brian Babler, for assistance with data acquisition and reduction.

This work has made use of the BeSS database, operated at GEPI, Observatoire de Meudon, France: http://basebe.obspm.fr. We would like to thank the amateur observers who obtained valuable spectra and generously made them available at the BeSS web page, namely to E. Barbotin, C. Buil, J. Guarroflo, B. Mauclaire, J. Ribeiro, J. Terry, O. Thizy and V. Desnoux. We thank Dr. D. Gies who provided us with their model derived from the CHARA observations, and the unknown referee for his/her very constructive comments and suggestions.

We thank the observers and staff at Ritter Observatory, especially Nancy Morrison, for their assistance in providing data used in this paper. Observations at Ritter Observatory are supported by the NSF under the PREST program, grant AST04-40784. J.P.W. is supported by a NSF Astronomy \& Astrophysics Postdoctoral Fellowship under award AST-0802230. The observations at Ondřejov Observatory were supported by the Grant Agency of the Academy of Sciences of the Czech Republic (grants AA3003001, AA 3003403). The Heros@Ondřjov monitoring project was part of a joint project supported by the German Bundesministerium für Bildung and Forschung and the Ministry of Education of the Czech Republic (TSE-001-009, 436 TSE 113/18 and 41). This work was also supported by FAPESP grant 04/07707-3 to A.C.C.

\section{References}

Bagnulo, S., Jehin, E. Ledoux, C., et al. 2003, ESO Messenger, 114, 10 Berio, P., Stee, P., Vakili, F., et al. 1999, A\&A, 345, 203

Bjorkman, K. S., \& Meade, M. R. 2005, in Astronomical Polarimetry: Current Status and Future Directions, ed. A. A. Adamson, C. Aspin, C. J. Davis, \& T. Fujiyoshi, ASP Conf. Ser., 343, 406

Božić, H., \& Pavlovski, K. 1988, Hvar Observatory Bulletin, 12, 15

Carciofi, A. C., \& Bjorkman, J. E. 2006, ApJ, 639, 1081

Carciofi, A. C., \& Bjorkman, J. E. 2008, ApJ, 684, 1374

Carciofi, A. C., Miroshnichenko, A. S., Kusakin, A. V. ., et al. 2006, ApJ, 652, 1617

Carciofi, A. C., Okazaki, A. T., le Bouquin, J.-B., et al. 2009, A\&A, 504, 915

Cushing, M. C., Vacca, W. D., \& Rayner, J. T. 2004, PASP, 116, 362
Doazan, V. 1987, in Physics of Be stars, ed. A. Slettebak, \& T. P. Snow, IAU Colloq., 92, 384

Doazan, V., \& Thomas, R. N. 1987, in Stellar pulsation, ed. A. N. Cox (SpringerVerlag), 96

Gies, D. R., Bagnuolo, Jr., W. G., Baines, E. K., et al. 2007, ApJ, 654, 527

Grosbøl, P. \& Ponz, D. 1990, in "The MIDAS System" in Acquisition, Processing and Archiving of Astronomical Images, ed. O. Longo \& G. Sedmak, OAC and FORMEZ, 109

Guo, Y., Huang, L., Hao, J., et al. 1995, A\&AS, 112, 201

Hanuschik, R. W. 1989, A\&A, 161, 61

Hanuschik, R. W. 1994, Ap\&SS, 216, 99

Hanuschik, R. W. 2000, in 214, IAU Colloq., 175: The Be Phenomenon in EarlyType Stars, ed. M. A. Smith, H. F. Henrichs, \& J. Fabregat, ASP Conf. Ser., 518

Hanuschik, R. W., Dachs, J., Baudzus, M., \& Thimm, G. 1993, A\&A, 274, 356

Harmanec, P. 1983, Hvar Observatory Bulletin, 7, 55

Harmanec, P. 1984, Bulletin of the Astronomical Institutes of Czechoslovakia, 35,164

Harmanec, P., Pavlovski, K., Božíc, H., et al. 1997, J. Astron. Data, 3, 5

Hirata, R. 2007, in Active OB-Stars: Laboratories for Stellare and Circumstellar Physics, ed. S. Štefl, S. P. Owocki, \& A. T. Okazaki, ASP Conf. Ser., 361, 267

Huang, S.-S. 1973, ApJ, 183, 541

Hummel, W. 1998, A\&A, 330, 243

Hummel, W. \& Hanuschik, R. W. 1997, A\&A, 320, 852

Hummel, W. \& Vrancken, M. 2000, A\&A, 359, 1075

Kato, S. 1983, PASJ, 35, 249

Kaufer, A. 1998, Rev. Mod. Astron., 11, 177

Kaufer, A., Stahl, O., Wolf, B., et al. 1996, A\&A, 305, 887

Kaufer, A., Stahl, O., Tubbesing, S., et al. 1999, ESO Messenger, 95, 8

Lachaume, R. 2003, A\&A, 400, 795

Lee, U., Osaki, Y., \& Saio, H. 1991, MNRAS, 250, 432

McDavid, D. 1999, PASP, 111, 494

McLean, I. S. \& Brown, J. C. 1978, A\&A, 69, 291

Mennickent, R. E. \& Vogt, N. 1991, A\&A, 241, 159

Mérand, A., Bordé, P., \& Coudé du Foresto, V. 2005, A\&A, 433, 1155

Millour, F., Petrov, R. G., Malbet, F., et al. 2007, in ESO Calibration Workshop 2007, 274

Nook, M. 1990, The envelopes of RV Tauri variables, Ph.D. Thesis Wisconsin Univ., Madison

Nordsieck, K., \& Harris, W. 1996, in Polarimetry of the interstellar medium, ed.

W. G. Roberge, \& D. C. B. Whittet, ASP Conf. Ser., 97, 100

Okazaki, A. T. 1991, PASJ, 43, 75

Okazaki, A. T. 1997, A\&A, 318, 548

Okazaki, A. T. 2000, in IAU Colloq. 175: The Be Phenomenon in Early-Type Stars, ed. M. A. Smith, H. F. Henrichs, \& J. Fabregat, ASP Conf. Ser., 214, 409

Okazaki, A. T. 2001, PASJ, 53, 119

Papaloizou, J. C. B. \& Savonije, G. J. 2006, A\&A, 456, 1097

Papaloizou, J. C. B., Savonije, G. J., \& Henrichs, H. F. 1992, A\&A, 265, L45

Pavlovski, K., Harmanec, P., Božić, H., et al. 1997, A\&AS, 125, 75

Petrov, R. G., Malbet, F., Weigelt, G., et al. 2007, A\&A, 464, 1

Poeckert, R. \& Marlborough, J. M. 1978, ApJS, 38, 229

Pollmann, E. \& Rivinius, T. 2008, IBVS, 5813, 1

Porter, J. M. 1996, MNRAS, 280, L31

Quirrenbach, A., Bjorkman, K. S., Bjorkman, J. E., et al. 1997, ApJ, 479, 477

Rayner, J. T., Toomey, D. W., Onaka, P. M., et al. 2006, PASP, 115, 362

Rivinius, T., Stefl, S., \& Baade, D. 1999, A\&A, 348, 831

Rivinius, T., Baade, D., \& Štefl, S. 2003, A\&A, 411, 229

Rivinius, T., Štefl, S., \& Baade, D. 2006, A\&A, 459, 137

Rousselot, P., Lidman, C., Cuby, J.-G., Moreels, G., \& Monnet, G. 2000, A\&A, 354,1134

Scargle, J. D. 1982, ApJ, 263, 835

Tatulli, E., Millour, F., Chelli, A., et al. 2007, A\&A, 464, 29

Tycner, C., Hajian, A. R., Armstrong, J. T., et al. 2004, ApJ, 127, 1194

Štefl, S., Okazaki, A. T., Rivinius, T., \& Baade, D. 2007, in Active OB-Stars: Laboratories for Stellar and Circumstellar Physics, ed. S. Štefl, S. P. Owocki, \& A. T. Okazaki, ASP Conf. Ser., 361, 274

Vacca, W. D., Cushing, M. C., \& Rayner, J. T. 2003, PASP, 115, 389

Vakili, F., Mourard, D., Stee, P., et al. 1998, A\&A, 335, 261

Waters, L. B. F. M. \& Marlborough, J. M. 1994, in Pulsation; Rotation; and Mass Loss in Early-Type Stars, ed. L. A. Balona, H. F. Henrichs, \& J. M. Le Contel, IAU Symp., 162, 399

Wisniewski, J. P. 2005, The Effect of Age and Metallicity on Be Circumstellar Disk Formation, Ph. D. Thesis Ritter Observatory, University of Toledo

Wisniewski, J. P., Kowalski, A. F., Bjorkman, K. S., Bjorkman, J. E., \& Carciofi, A. C. 2007, ApJ, 656, L21

Wolff, M., Nordsieck, K., \& Nook, M. 1996, AJ, 111, 856 